\title{
REVIEW
}

\section{Physiological changes after fluid bolus therapy in sepsis: a systematic review of contemporary data}

Neil J Glassford ${ }^{1,2}$, Glenn M Eastwood ${ }^{1,3}$ and Rinaldo Bellomo ${ }^{1,2^{*}}$

\begin{abstract}
Fluid bolus therapy (FBT) is a standard of care in the management of the septic, hypotensive, tachycardic and/or oliguric patient. However, contemporary evidence for FBT improving patient-centred outcomes is scant. Moreover, its physiological effects in contemporary ICU environments and populations are poorly understood. Using three electronic databases, we identified all studies describing FBT between January 2010 and December 2013. We found 33 studies describing 41 boluses. No randomised controlled trials compared FBT with alternative interventions, such as vasopressors. The median fluid bolus was $500 \mathrm{ml}$ (range 100 to 1,000 ml) administered over 30 minutes (range 10 to 60 minutes) and the most commonly administered fluid was $0.9 \%$ sodium chloride solution. In 19 studies, a predetermined physiological trigger initiated FBT. Although 17 studies describe the temporal course of physiological changes after FBT in 31 patient groups, only three studies describe the physiological changes at 60 minutes, and only one study beyond this point. No studies related the physiological changes after FBT with clinically relevant outcomes. There is a clear need for at least obtaining randomised controlled evidence for the physiological effects of FBT in patients with severe sepsis and septic shock beyond the period immediately after its administration.

Just as water retains no shape, so in warfare there are no constant conditions

Sun Tzu (The Art of War)
\end{abstract}

\section{Introduction}

All critically ill patients receive intravenous (IV) fluids, which are given to maintain physiological homeostasis, or as a vehicle for drug administration, or as direct therapeutic administration to correct perceived haemodynamic instability [1-4]. In these situations, where there is a perceived reduction in venous return and cardiac output secondary to vasodilatation and/or hypovolaemia, using IV fluid to increase intravascular volume is believed to effectively compensate for these changes in vascular tone by increasing stroke volume in accordance with the Frank-Starling principle [5-10].

Several mechanisms for delivering IV fluids, both diagnostically and therapeutically under such circumstances, have been described. These include Weil s central venous pressure (CVP)-guided fluid challenge technique [10-13],

\footnotetext{
* Correspondence: rinaldo.bellomo@austin.org.au

'Department of Intensive Care, Austin Hospital, Melbourne, Victoria 3084, Australia

${ }^{2}$ Australian and New Zealand Intensive Care Research Centre, School of Public Health and Preventive Medicine, Monash University, Melbourne, Victoria 3004, Australia

Full list of author information is available at the end of the article
}

the timed and rapid infusion methods favoured by Shoemaker [7,8,14-16] and, more recently, techniques involving echocardiographic or ultrasonographic assessment of fluid responsiveness following low-volume IV infusion [17]. However, the current standard of care in the management of septic, hypotensive, tachycardic and/or oliguric patients is fluid bolus therapy (FBT), where IV fluid is rapidly administered in discrete boluses [18-21]. While the ideal fluid bolus would be a discrete volume of a specific fluid administered at a specified rate, accounting for individual patient features and with a defined aim (Figure 1) [11], there is no current agreement regarding exactly what defines a fluid bolus. Moreover, although strong overall consensus regarding the importance of FBT exists [18-20], there appears to be little randomized controlled information on the magnitude and duration of its physiological effects, or on the direct positive impact of FBT on patient outcome in sepsis as an independent intervention [22].

In contrast, an expanding body of evidence suggests that FBT may contribute to a positive fluid balance, which, in turn, is independently associated with a variety of adverse outcomes in the critically ill [23-28]. Recent

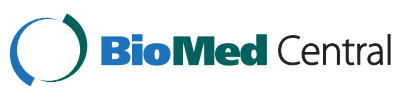

2014 Glassford et al.; licensee BioMed Central Ltd. The licensee has exclusive rights to distribute this article, in any medium, for 12 months following its publication. After this time, the article is available under the terms of the Creative Commons Attribution License (http://creativecommons.org/licenses/by/4.0), which permits unrestricted use, distribution, and reproduction in any medium, provided the original work is properly credited. The Creative Commons Public Domain Dedication waiver (http://creativecommons.org/publicdomain/zero/1.0/) applies to the data made available in this article, unless otherwise stated. 


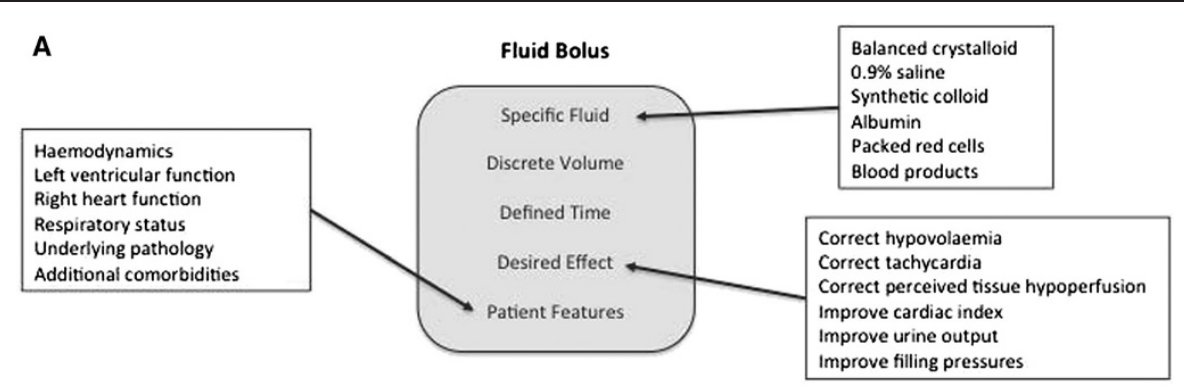

B

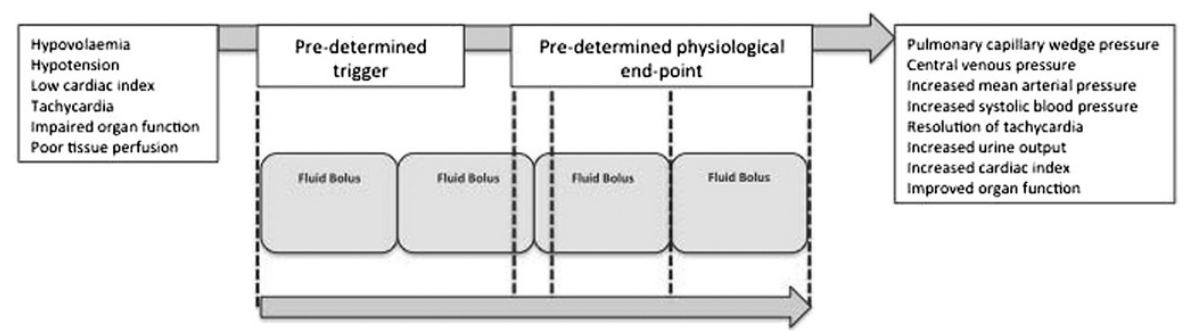

Figure 1 Describing the concept of idealised fluid bolus therapy. (A) Diagram describing the key criteria defining the concept of a fluid bolus. (B) Diagram describing the idealised concept of fluid bolus therapy in critical care, including purpose, triggers, end-points and purported physiological effects of such resuscitation.

experimental evidence suggests rapid fluid infusion can also damage the endothelial glycocalyx $[29,30]$, a structure already at risk in patients with sepsis [31], leading to endothelial disruption and organ dysfunction $[32,33]$. It appears that we need a better understanding of both the current evidence base for FBT and how best to apply it in the clinical setting [34,35].

Accordingly, we systematically reviewed the contemporary literature to determine current practice and to identify the independent effects of FBT on both physiological and patient-centred outcomes in the management of severe sepsis and septic shock in critical care practice.

\section{Methods}

We interrogated the MEDLINE, CENTRAL and EMBASE electronic reference databases using a combination of search terms (Figure 2). The reference lists of retrieved articles were examined for additional studies of potential relevance.

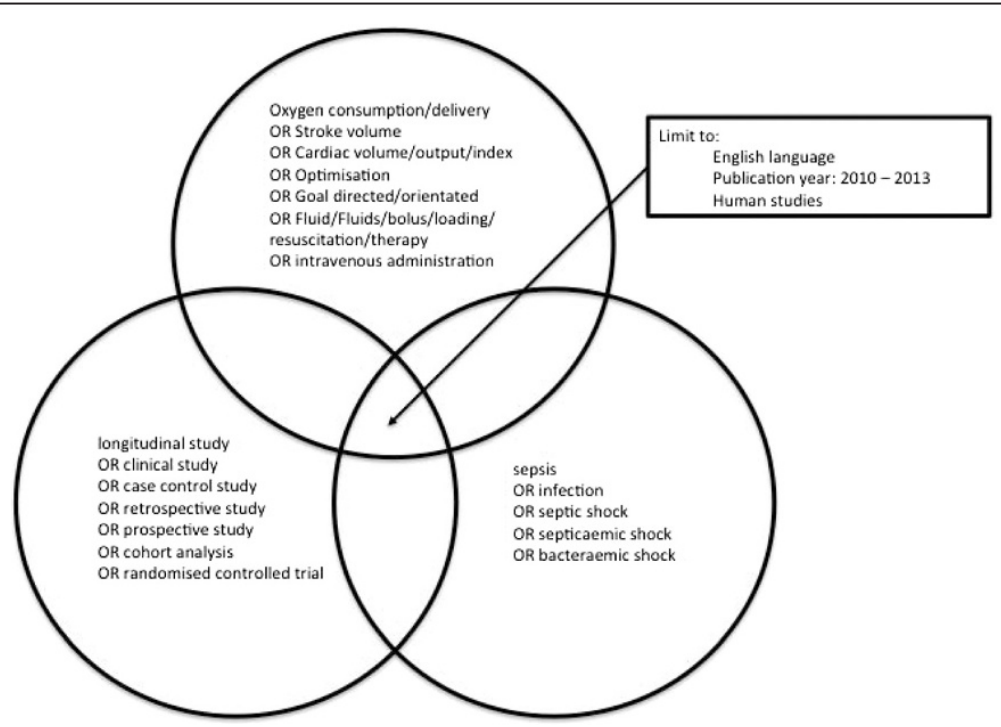

Figure 2 Electronic search strategy. Diagrammatic representation of the search strategy combining terms representing fluid resuscitation, sepsis and clinical studies, along with predetermined limitations. 
The search was carried out in December 2013. To achieve contemporary relevance results were arbitrarily limited to this decade (2010 to 2013) and to English language studies in humans. Paediatric studies were excluded. This search defined a set of records of studies of fluid administration or haemodynamic optimization in patients with severe sepsis or septic shock.

The abstracts of these records were examined to identify those studies of potential relevance. These manuscripts were retrieved and examined manually in accordance with our inclusion criteria. The studies to be included in the review were checked to ensure they had not been retracted subsequent to their publication.

\section{Study inclusion criteria}

\section{Population of included studies}

We considered clinical studies of any type describing a population of patients suffering from severe sepsis or septic shock. We also included those studies of shock or circulatory failure where either the majority of patients, or a defined subgroup of patients, had severe sepsis or septic shock.

\section{Intervention - fluid bolus administration}

For the purposes of this study a fluid bolus was a defined volume of a defined fluid administered over a defined time period. We recognised that most studies do not describe FBT in ideal terms (Figure 1) and therefore studies describing at least two of the three criteria were included in the review.

\section{Comparator - alternatives to fluid administration}

Any studies comparing FBT with the initiation of vasoactive medication, the increase of such medication or observation as an alternative to the administration of FBT were included in the review.

\section{Between groups analysis}

Where studies included in the review assigned patients to multiple treatment arms, each treatment group was treated as an individual group.

\section{Outcome - physiological effects of bolus administration}

Subsets of studies were selected from those describing FBT. The first included those reporting changes in cardiac output, heart rate, mean arterial pressure, central venous pressure, venous oxygen saturation, blood lactate concentration, urine output or haemoglobin concentration following FBT; for the purposes of inclusion, studies could describe changes in any or all of the haemodynamic parameters listed, but the direction, magnitude and duration of the change had to be extractable from tables or figures contained in the paper. The second group included those reporting non-physiological, patient-centred outcomes. Our primary outcome of interest was mortality at all reported time points. Secondary outcomes of interest included duration of ICU and hospital stay, duration of mechanical ventilation, and need for continuous renal replacement therapy (CRRT). We did not contact authors for additional information or individual patient data.

\section{Data collection}

We collected data on study type, study setting and location, study population and the aims of the study. Due to our acceptance of multiple types of study, we chose not to adopt a methodological scoring system. We examined the definition of a fluid bolus in each study fulfilling our criteria and recorded the type and volume of fluid used, as well as the rate of administration. We identified the trigger and end-points for fluid bolus administration, the number of boluses administered and the use of red cell transfusions and vasoactive medication as part of the experimental protocol. We identified the demographic group in which subsequent observations were recorded. In those studies describing the physiological effects of bolus administration, we recorded the absolute change in cardiac output, heart rate, mean arterial pressure, venous oxygen saturation, blood lactate concentration, urine output and haemoglobin concentration. In those studies reporting patient-centred outcomes we recorded mortality at all reported time points, duration of ICU and hospital stay, duration of mechanical ventilation, and need for CRRT.

\section{Statistical analysis}

We expected grossly heterogeneous results across different study types and study protocols. A meta-analysis approach could not be applied. Results are therefore presented as crude medians with full ranges. These exclude alternative units of measure, which are reported separately - for example, the median may be given in millilitres, followed by individual reporting of $\mathrm{ml} / \mathrm{kg}$.

\section{Results}

\section{Electronic search}

Our search strategy identified 2,956 articles over the period 2010 to 2013. Of these, 2,875 were excluded as duplicates, irrelevant, paediatric research or having been published in a language other than English. Of the 81 potentially relevant publications identified, 33 met our inclusion criteria (Figure 3) [36-68]. In total, 17 of these described the physiological changes occurring following FBT $[36,39,40,45,46,48,50,53-55,57,59,60,62,63,65,66]$ and seven studies described patient-orientated outcome measures $[37,42,43,49,58,59,64]$.

\section{Relevant contemporary studies}

The study details, population, size and aims are presented in Table 1 . We identified 22 prospective observational 


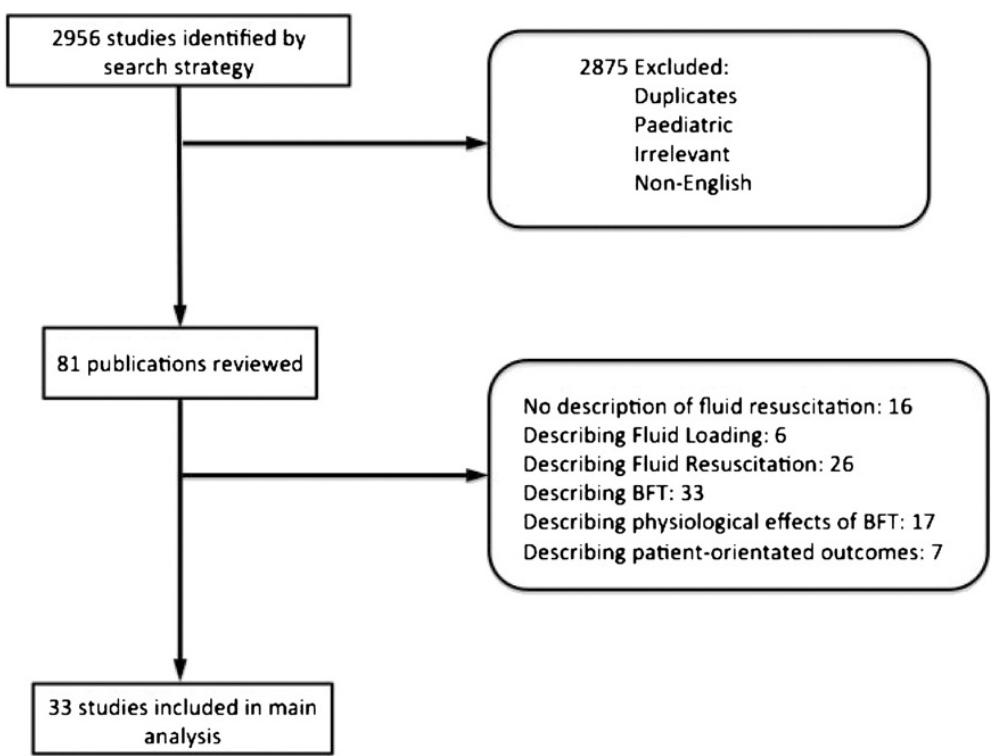

Figure 3 Study selection. Flow diagram of the study selection process and detailed description of study exclusions. FBT, fluid bolus therapy.

studies, four retrospective observational studies, two quasi-experimental studies, and five randomised controlled trials (RCTs). Of the five RCTs, none compared FBT with a control intervention; two actually reported the impact of blood volume analysis on protocolized resuscitation [64,67]; two compared hypertonic versus isotonic fluids $[51,65]$; and one actually compared two vasopressors and reported fluid data as an addendum [38]. Additional study data can be found in the electronic supplemental material (Additional file 1: Table S1).

\section{Pre-fluid bolus therapy fluid administration}

Fluid resuscitation prior to study recruitment and FBT was described in 10 studies. In the five studies describing finite volumes of resuscitation fluid, the median volume administered was $2,200 \mathrm{ml}$ (range 1,000 to $5,060 \mathrm{ml}$ ) $[38,47,51,53,58]$. The five remaining studies reported weight-dependent volumes of between 20 and $30 \mathrm{ml} / \mathrm{kg}$ of resuscitation (Table 2) $[41,43,49,56,57]$.

\section{Initiation and cessation of fluid bolus therapy}

Across the 33 studies, 19 predetermined clinical or physiological features triggered FBT. In the remaining 14 studies, FBT was triggered by clinical judgment in eight, by hypotension in two, simply by the diagnosis of severe sepsis or septic shock in two, and remained unspecified in two (Table 2).

In the majority of studies (18 of 33) FBT ceased at the end of the bolus in question; 10 studies used predetermined immediate changes in physiological variables as end-points; four studies did not define the physiological end-points of fluid resuscitation (Table 2).

\section{Defining fluid bolus therapy}

Overall, 41 forms of FBT were described, fully or in part, in 33 studies. They are presented in Table 2. In 20 studies, the fluid type was fixed; in 13 more than one fluid type was used. In six studies the fluid type was not identified beyond the generic crystalloid or colloid. The fluid most commonly used as a bolus was $0.9 \%$ saline (17 studies), followed by $6 \%$ hydroxyethyl starch (eight studies). On the other hand, $4 \%$ albumin was used in only four studies $[38,53,59,65], 4 \%$ gelatin in only three $[38,48,66]$, physiological lactated solutions in only two [59,61], and $20 \%$ albumin and blood products in only one [38].

The median amount of fluid administered as a finite volume was $500 \mathrm{ml}$ (range 100 to $1,000 \mathrm{ml}$ ). However, $20 \mathrm{ml} / \mathrm{kg}$ and $7 \mathrm{ml} / \mathrm{kg}$ were individually reported as weight-dependent boluses. The median number of boluses (24 studies) was 1 (range 0.68 to 10 ). Rates of administration were defined for 31 of 41 boluses with a median rate of 30 minutes (range 10 to 60 minutes).

\section{Haemodynamic changes after fluid bolus therapy Comparing different interventions}

No RCTs compared the haemodynamic changes induced by FBT with observation or vasopressor administration or inotropic drug administration or continuous low dose IV fluid infusion or any combination of the above. The only study comparing FBT with an alternative intervention was a single, non-randomized, prospective, observational study that compared acute circulatory failure patients treated with FBT (500 $\mathrm{ml}$ of saline) or with increased norepinephrine dose according to clinician preference [55]. The two groups had clearly different baseline characteristics and were not directly compared. 
Table 1 Study settings, size, population and aims

\begin{tabular}{|c|c|c|c|c|c|c|c|}
\hline First author & Journal & Year & Aims of study & Location & Institution(s) & Study type & Population size \\
\hline Bihari [36] & Shock & 2013 & $\begin{array}{l}\text { Investigation of the use and effects } \\
\text { of fluid boluses in septic patients } \\
\text { following primary resuscitation }\end{array}$ & Australia & $\begin{array}{l}\text { Single centre, } \\
\text { academic ICU }\end{array}$ & $\begin{array}{l}\text { Prospective } \\
\text { observational } \\
\text { study }\end{array}$ & $\begin{array}{l}50 \text { patients with severe sepsis } \\
\text { or septic shock }\end{array}$ \\
\hline Castellanos-Ortega [37] & Critical Care Medicine & 2010 & $\begin{array}{l}\text { Evaluation of the impact of a } \\
\text { standardised EGDT response to sepsis }\end{array}$ & Spain & $\begin{array}{l}\text { Single centre, } \\
\text { academic ICU }\end{array}$ & $\begin{array}{l}\text { Quasi-experimental } \\
\text { study }\end{array}$ & 480 patients with septic shock \\
\hline De Backer [38] & $\begin{array}{l}\text { New England Journal } \\
\text { of Medicine }\end{array}$ & 2010 & $\begin{array}{l}\text { Assessing the effect of noradrenaline } \\
\text { as first-line vasopressor on mortality }\end{array}$ & Europe & $\begin{array}{l}8 \text { centres, } \\
\text { mixed ICUs }\end{array}$ & $\begin{array}{l}\text { Randomised } \\
\text { clinical trial }\end{array}$ & $\begin{array}{l}1,679 \text { patients with shock requiring } \\
\text { vaspressor therapy. 1,044 patients } \\
\text { with sepsis }\end{array}$ \\
\hline Dong [39] & $\begin{array}{l}\text { World Journal of } \\
\text { Emergency Medicine }\end{array}$ & 2012 & $\begin{array}{l}\text { Investigating the relationship } \\
\text { between stroke volume index } \\
\text { and passive leg raising and fluid } \\
\text { responsiveness }\end{array}$ & China & $\begin{array}{l}2 \text { centres, } \\
\text { general ICUs }\end{array}$ & $\begin{array}{l}\text { Prospective } \\
\text { observational } \\
\text { study }\end{array}$ & $\begin{array}{l}32 \text { mechanically ventilated patients } \\
\text { with septic shock }\end{array}$ \\
\hline Freitas [40] & British Journal of Anaesthesia & 2013 & $\begin{array}{l}\text { Evaluation of the predictive value } \\
\text { of automated PPV for fluid } \\
\text { responsiveness in patients with } \\
\text { sepsis and low tidal volumes }\end{array}$ & Brazil & $\begin{array}{l}\text { Single centre, } \\
\text { academic ICU }\end{array}$ & $\begin{array}{l}\text { Prospective } \\
\text { observational } \\
\text { study }\end{array}$ & $\begin{array}{l}40 \text { patients with low tidal volume } \\
\text { ventilation and severe sepsis or septic } \\
\text { shock requiring a fluid challenge }\end{array}$ \\
\hline Gaieski [41] & Critical Care Medicine & 2010 & $\begin{array}{l}\text { Evaluation of the impact of a } \\
\text { standardised EGDT response to } \\
\text { sepsis on time to antibiotic } \\
\text { administration and survival }\end{array}$ & USA & $\begin{array}{l}\text { Single centre, } \\
\text { academic ICU }\end{array}$ & $\begin{array}{l}\text { Retrospective } \\
\text { observational } \\
\text { study }\end{array}$ & $\begin{array}{l}261 \text { patients with severe sepsis and } \\
\text { septic shock undergoing EGDT }\end{array}$ \\
\hline Hamzaoui [42] & Critical Care & 2010 & $\begin{array}{l}\text { Evaluation of the cardiac } \\
\text { consequences of early } \\
\text { administration of noradrenaline }\end{array}$ & France & $\begin{array}{l}\text { Single centre, } \\
\text { academic ICU }\end{array}$ & $\begin{array}{l}\text { Prospective } \\
\text { observational } \\
\text { study }\end{array}$ & $\begin{array}{l}105 \text { patients with septic shock } \\
\text { requiring vasopressor commencement } \\
\text { following initial fluid resuscitation }\end{array}$ \\
\hline Hanzelka [43] & $\begin{array}{l}\text { Supportive Care in } \\
\text { Cancer }\end{array}$ & 2013 & $\begin{array}{l}\text { Evaluation of the impact of a } \\
\text { standardised EGDT response } \\
\text { to sepsis }\end{array}$ & USA & $\begin{array}{l}\text { Single centre, } \\
\text { academic ED }\end{array}$ & $\begin{array}{l}\text { Retrospective } \\
\text { observational } \\
\text { study }\end{array}$ & $\begin{array}{l}200 \text { patients with cancer and severe } \\
\text { sepsis or septic shock presenting to ED }\end{array}$ \\
\hline Jacob [44] & Critical Care Medicine & 2012 & $\begin{array}{l}\text { Evaluation of the impact of } \\
\text { early monitored sepsis } \\
\text { management }\end{array}$ & Uganda & $\begin{array}{l}2 \text { centres, } \\
\text { medical/treatment } \\
\text { centres }\end{array}$ & $\begin{array}{l}\text { Prospective } \\
\text { observational } \\
\text { study }\end{array}$ & $\begin{array}{l}671 \text { patients with severe sepsis } \\
\text { presenting within office hours }\end{array}$ \\
\hline Khwannimit [45] & $\begin{array}{l}\text { European Journal of } \\
\text { Anaesthesiology }\end{array}$ & 2012 & $\begin{array}{l}\text { Comparing SW by Vigileo with } \\
\text { PPV by monitor to predict } \\
\text { fluid responsiveness }\end{array}$ & Thailand & $\begin{array}{l}\text { Single centre, } \\
\text { academic ICU }\end{array}$ & $\begin{array}{l}\text { Prospective } \\
\text { observational } \\
\text { study }\end{array}$ & $\begin{array}{l}42 \text { patients with septic shock who } \\
\text { were mechanically ventilated with } \\
\text { tidal volumes }>8 \mathrm{ml} / \mathrm{kg} \text { requiring } \\
\text { fluid resuscitation }\end{array}$ \\
\hline Lakhal [46] & Intensive Care Medicine & 2013 & $\begin{array}{l}\text { Identification of fluid } \\
\text { responsiveness from IABP } \\
\text { and NIBP }\end{array}$ & France & $\begin{array}{l}3 \text { centres, } \\
\text { academic ICU }\end{array}$ & $\begin{array}{l}\text { Prospective } \\
\text { observational } \\
\text { study }\end{array}$ & $\begin{array}{l}130 \text { patients with circulatory failure } \\
\text { requiring a fluid challenge. } 58 \\
\text { patients with septic shock }\end{array}$ \\
\hline Lanspa [47] & Journal of Critical Care & 2012 & $\begin{array}{l}\text { Assessment of CVP and shock } \\
\text { index to predict haemodynamic } \\
\text { response to volume expansion } \\
\text { when compared with CVP alone }\end{array}$ & USA & $\begin{array}{l}\text { Single centre, } \\
\text { academic ICU }\end{array}$ & $\begin{array}{l}\text { Prospective } \\
\text { observational } \\
\text { study }\end{array}$ & $\begin{array}{l}25 \text { patients with septic shock over } \\
14 \text { years of age }\end{array}$ \\
\hline
\end{tabular}


Table 1 Study settings, size, population and aims (Continued)

\begin{tabular}{|c|c|c|c|c|c|c|c|}
\hline Machare-Delgado [48] & $\begin{array}{l}\text { Journal of Intensive } \\
\text { Care Medicine }\end{array}$ & 2011 & $\begin{array}{l}\text { Predicting fluid responsiveness } \\
\text { by comparing SW and inferior } \\
\text { vena caval respiratory variation } \\
\text { by ECHO during mechanical } \\
\text { ventilation }\end{array}$ & USA & $\begin{array}{l}\text { Single centre, } \\
\text { medical } \\
\text { academic ICU }\end{array}$ & $\begin{array}{l}\text { Prospective } \\
\text { observational } \\
\text { study }\end{array}$ & $\begin{array}{l}25 \text { mechanically ventilated } \\
\text { vasopressor-dependent patients } \\
\text { who required a fluid challenge. } \\
22 \text { patients with severe sepsis or } \\
\text { septic shock }\end{array}$ \\
\hline MacRedmond [49] & $\begin{array}{l}\text { Quality and Safety } \\
\text { in Health Care }\end{array}$ & 2010 & $\begin{array}{l}\text { Evaluation of the impact of } \\
\text { implementing a quality initiative } \\
\text { on the management of severe } \\
\text { sepsis and septic shock }\end{array}$ & Canada & Single centre, ICU & $\begin{array}{l}\text { Quasi-experimental } \\
\text { study }\end{array}$ & $\begin{array}{l}74 \text { patients with severe sepsis or } \\
\text { septic shock admitted via ED }\end{array}$ \\
\hline Mahjoub [50] & Intensive Care Medicine & 2012 & $\begin{array}{l}\text { Assessment of the impact of } \\
\text { volume expansion on patients } \\
\text { with left ventricular dysfunction }\end{array}$ & France & $\begin{array}{l}\text { Single centre, } \\
\text { academic ICU }\end{array}$ & $\begin{array}{l}\text { Prospective } \\
\text { observational } \\
\text { study }\end{array}$ & $\begin{array}{l}83 \text { mechanically ventilated patients } \\
\text { with sepsis-induced circulatory failure }\end{array}$ \\
\hline Mclntyre [51] & Journal of Critical Care & 2012 & $\begin{array}{l}\text { Feasibility study comparing the } \\
\text { effects of } 5 \% \text { albumin versus } 0.9 \% \\
\text { saline for resuscitation in septic shock }\end{array}$ & Canada & $\begin{array}{l}6 \text { centres, } \\
\text { academic } \\
\text { ED and ICU }\end{array}$ & $\begin{array}{l}\text { Randomised } \\
\text { clinical trial }\end{array}$ & $\begin{array}{l}50 \text { patients with refractory hypotension } \\
\text { and sepsis }\end{array}$ \\
\hline Monnet [52] & Critical Care & 2010 & $\begin{array}{l}\text { Comparing haemodynamic changes } \\
\text { induced by noradrenaline and volume } \\
\text { expansion using Vigileo and PiCCO }\end{array}$ & France & $\begin{array}{l}\text { Single centre, } \\
\text { academic } \\
\text { medical ICU }\end{array}$ & $\begin{array}{l}\text { Prospective } \\
\text { observational } \\
\text { study }\end{array}$ & $\begin{array}{l}80 \text { patients with sepsis-induced } \\
\text { circulatory failure }\end{array}$ \\
\hline Monnet [53] & Critical Care Medicine & 2011 & $\begin{array}{l}\text { Assessing the effects of noradrenaline } \\
\text { on haemodynamics in sepsis }\end{array}$ & France & $\begin{array}{l}\text { Single centre, } \\
\text { academic } \\
\text { medical ICU }\end{array}$ & $\begin{array}{l}\text { Prospective } \\
\text { observational } \\
\text { study }\end{array}$ & $\begin{array}{l}25 \text { patients with sepsis-induced } \\
\text { fluid-responsive acute circulatory } \\
\text { failure with } \mathrm{DBP}<40 \mathrm{mmHg} \text {, or } \\
\text { requiring noradrenaline }\end{array}$ \\
\hline Monnet [54] & Critical Care Medicine & 2013 & $\begin{array}{l}\text { Comparing } \mathrm{ScvO}_{2} \text { and markers of } \\
\text { anaerobic metabolism as predictors } \\
\text { of unfavourable changes in oxygen } \\
\text { extraction }\end{array}$ & France & $\begin{array}{l}\text { Single centre, } \\
\text { academic } \\
\text { medical ICU }\end{array}$ & $\begin{array}{l}\text { Prospective } \\
\text { observational } \\
\text { study }\end{array}$ & $\begin{array}{l}51 \text { patients with acute circulatory } \\
\text { failure undergoing transpulmonary } \\
\text { thermodilution monitoring, } 40 \\
\text { patients with septic shock }\end{array}$ \\
\hline Monnet [55] & Critical Care Medicine & 2011 & $\begin{array}{l}\text { Investigation of the utility of pulse } \\
\text { pressure as a surrogate for changes } \\
\text { in cardiac output }\end{array}$ & France & $\begin{array}{l}\text { Single centre, } \\
\text { academic } \\
\text { medical ICU }\end{array}$ & $\begin{array}{l}\text { Prospective } \\
\text { observational } \\
\text { study }\end{array}$ & $\begin{array}{l}373 \text { patients with acute circulatory } \\
\text { failure requiring a fluid challenge or } \\
\text { the introduction or dose increase } \\
\text { of noradrenaline. } 338 \text { patients } \\
\text { with septic shock }\end{array}$ \\
\hline O Neill [56] & $\begin{array}{l}\text { Journal of Emergency } \\
\text { Medicine }\end{array}$ & 2012 & $\begin{array}{l}\text { Evaluation of the most difficult } \\
\text { elements of a SSC protocol to } \\
\text { implement in a community-based ED }\end{array}$ & USA & $\begin{array}{l}\text { Single centre, } \\
\text { community ED }\end{array}$ & $\begin{array}{l}\text { Retrospective } \\
\text { observational } \\
\text { study }\end{array}$ & $\begin{array}{l}79 \text { with severe sepsis or septic } \\
\text { shock remaining hypotensive } \\
\text { following } 2,000 \mathrm{ml} \text { of fluid } \\
\text { resuscitation }\end{array}$ \\
\hline Ospina-Tascon [57] & Intensive Care Medicine & 2010 & $\begin{array}{l}\text { Evaluation of the effects of fluid } \\
\text { administration on microcirculatory } \\
\text { alterations in sepsis }\end{array}$ & Belgium & $\begin{array}{l}\text { Single centre, } \\
\text { academic ICU }\end{array}$ & $\begin{array}{l}\text { Prospective } \\
\text { observational } \\
\text { study }\end{array}$ & $\begin{array}{l}60 \text { patients with severe sepsis } \\
\text { requiring fluid challenge. } 37 \\
\text { within } 24 \text { hours of diagnosis, } \\
23 \text { after } 48 \text { hours }\end{array}$ \\
\hline Patel [58] & $\begin{array}{l}\text { Annals of } \\
\text { Pharmacotherapy }\end{array}$ & 2010 & $\begin{array}{l}\text { Investigation of the implementation } \\
\text { and effects of introducing the } \\
\text { SSC guidelines }\end{array}$ & USA & $\begin{array}{l}\text { Single centre, } \\
\text { community ICU }\end{array}$ & $\begin{array}{l}\text { Prospective } \\
\text { observational } \\
\text { study }\end{array}$ & $\begin{array}{l}112 \text { patients with sepsis or } \\
\text { septic shock }\end{array}$ \\
\hline
\end{tabular}


Table 1 Study settings, size, population and aims (Continued)

\begin{tabular}{|c|c|c|c|c|c|c|c|}
\hline Pierrakos [59] & Intensive Care Medicine & 2012 & $\begin{array}{l}\text { Evaluation of the correlation } \\
\text { between changes in MAP and } \\
\text { Cl following fluid challenge }\end{array}$ & Belgium & $\begin{array}{l}\text { Single centre, } \\
\text { academic ICU }\end{array}$ & $\begin{array}{l}\text { Prospective } \\
\text { observational } \\
\text { study }\end{array}$ & $\begin{array}{l}51 \text { patients with septic shock } \\
\text { undergoing invasive haemodynamic } \\
\text { monitoring and requiring a fluid } \\
\text { challenge }\end{array}$ \\
\hline Pottecher [60] & Intensive Care Medicine & 2010 & $\begin{array}{l}\text { Assessment of sublingual } \\
\text { microcirculatory changes in } \\
\text { response to fluid challenge }\end{array}$ & France & $\begin{array}{l}2 \text { centres, } \\
\text { academic ED }\end{array}$ & $\begin{array}{l}\text { Prospective } \\
\text { observational } \\
\text { study }\end{array}$ & $\begin{array}{l}25 \text { mechanically ventilated patients } \\
\text { with severe sepsis or septic shock } \\
\text { within } 24 \text { hours of ICU admission } \\
\text { demonstrating pre-load dependency }\end{array}$ \\
\hline Sanchez [61] & $\begin{array}{l}\text { Anaesthesia and } \\
\text { Intensive Care }\end{array}$ & 2011 & $\begin{array}{l}\text { Measuring the response to a } \\
\text { fluid load in patients with and } \\
\text { without septic shock }\end{array}$ & Spain & $\begin{array}{l}\text { Single centre, } \\
\text { academic ICU }\end{array}$ & $\begin{array}{l}\text { Prospective } \\
\text { observational } \\
\text { study }\end{array}$ & $\begin{array}{l}32 \text { patients requiring invasive } \\
\text { monitoring. } 18 \text { patients with } \\
\text { septic shock }\end{array}$ \\
\hline Schnell [62] & Critical Care Medicine & 2013 & $\begin{array}{l}\text { Assessment of the effects of a } \\
\text { fluid challenge on Doppler-based } \\
\text { renal resistive index in critically ill } \\
\text { patients }\end{array}$ & France & $\begin{array}{l}3 \text { centres, } \\
\text { academic ICUs }\end{array}$ & $\begin{array}{l}\text { Prospective } \\
\text { observational } \\
\text { study }\end{array}$ & $\begin{array}{l}35 \text { mechanically ventilated } \\
\text { patients with real-time cardiac } \\
\text { monitoring requiring a fluid } \\
\text { challenge. } 30 \text { patients with sepsis }\end{array}$ \\
\hline Sturgess [63] & $\begin{array}{l}\text { Anaesthesia and } \\
\text { Intensive Care }\end{array}$ & 2010 & $\begin{array}{l}\text { Comparison of aortic corrected } \\
\text { flow time, BNP and CVP as } \\
\text { predictors of fluid responsiveness }\end{array}$ & Australia & $\begin{array}{l}\text { Single centre, } \\
\text { private ICU }\end{array}$ & $\begin{array}{l}\text { Prospective } \\
\text { observational } \\
\text { study }\end{array}$ & $\begin{array}{l}10 \text { patients with septic shock } \\
\text { requiring a fluid challenge }\end{array}$ \\
\hline Trof [64] & Critical Care Medicine & 2012 & $\begin{array}{l}\text { Comparison of volume-guided } \\
\text { and pressure-guided hemodynamic } \\
\text { management in shocked patients }\end{array}$ & Netherlands & $\begin{array}{l}2 \text { centres, } \\
\text { academic, ICU }\end{array}$ & $\begin{array}{l}\text { Randomised } \\
\text { clinical trial }\end{array}$ & $\begin{array}{l}120 \text { patients with shock requiring } \\
\text { invasive haemodynamic monitoring } \\
\text { and }>48 \text { hours of ICU admission. } \\
72 \text { patients with sepsis }\end{array}$ \\
\hline van Haren [65] & Shock & 2012 & $\begin{array}{l}\text { Evaluation of the effects of hypertonic } \\
\text { versus isotonic fluid administration } \\
\text { in patients with septic shock }\end{array}$ & Netherlands & $\begin{array}{l}\text { Single centre, } \\
\text { academic ICU }\end{array}$ & $\begin{array}{l}\text { Randomised } \\
\text { clinical trial }\end{array}$ & $\begin{array}{l}24 \text { patients with septic shock } \\
\text { enrolled within } 24 \text { hours of } \\
\text { admission }\end{array}$ \\
\hline Wacharasint [66] & $\begin{array}{l}\text { Journal of the Medical } \\
\text { Association of Thailand }\end{array}$ & 2012 & $\begin{array}{l}\text { Evaluation of the effectiveness of } \\
\text { three dynamic measures of fluid } \\
\text { responsiveness in septic shock patients }\end{array}$ & Thailand & $\begin{array}{l}\text { Single centre, } \\
\text { medical ICU }\end{array}$ & $\begin{array}{l}\text { Prospective } \\
\text { observational } \\
\text { study }\end{array}$ & $\begin{array}{l}20 \text { patients with sepsis and acute } \\
\text { circulatory failure with invasive } \\
\text { haemodynamic monitoring stable } \\
\text { for } 15 \text { minutes prior to inclusion }\end{array}$ \\
\hline Yu [67] & Shock & 2011 & $\begin{array}{l}\text { Evaluation of the effects of blood } \\
\text { volume analysis compared with } \\
\text { pulmonary artery catheter monitoring }\end{array}$ & North America & $\begin{array}{l}\text { Single centre, } \\
\text { academic ICU }\end{array}$ & $\begin{array}{l}\text { Randomised } \\
\text { clinical trial }\end{array}$ & $\begin{array}{l}100 \text { patients requiring resuscitation } \\
\text { for shock. } 69 \text { patients with severe } \\
\text { sepsis or septic shock }\end{array}$ \\
\hline Zhang [68] & Journal of Critical Care & 2012 & $\begin{array}{l}\text { Investigation of the association } \\
\text { between plasma protein levels } \\
\text { and subsequent pulmonary oedema }\end{array}$ & China & $\begin{array}{l}\text { Single centre, } \\
\text { academic ICU }\end{array}$ & $\begin{array}{l}\text { Retrospective } \\
\text { observational } \\
\text { study }\end{array}$ & $\begin{array}{l}62 \text { patients with sepsis undergoing } \\
\text { transpulmonary thermodilution } \\
\text { assessment requiring fluid }\end{array}$ \\
\hline
\end{tabular}

BNP, B-type natriuretic peptide; $\mathrm{Cl}$, cardiac index; CVP, central venous pressure; DBP, diastolic blood pressure; ECHO, echocardiogram; ED, Emergency Department; EGDT, early goal directed therapy; IABP, intra-arterial blood pressure; MAP, mean arterial blood pressure; NIBP, non-invasive blood pressure; $\mathrm{PiCCO}$, pulse contour cardiac output monitoring; $\mathrm{PPV}$, pulse pressure variation; $\mathrm{ScvO}_{2}$, central venous oxygen saturation; $\mathrm{SSC}$, Surviving Sepsis Campaign; SVV, stroke volume variation. 
Table 2 Description of fluid boluses, triggers, physiological end-points and primary confounders

\begin{tabular}{|c|c|c|c|c|c|c|c|c|c|c|}
\hline First author & Year & $\begin{array}{l}\text { Initial } \\
\text { resuscitation }\end{array}$ & $\begin{array}{l}\text { Bolus fluid } \\
\text { type }\end{array}$ & $\begin{array}{l}\text { Bolus fluid } \\
\text { volume (ml) }\end{array}$ & $\begin{array}{l}\text { Bolus fluid } \\
\text { rate (minutes) }\end{array}$ & $\begin{array}{l}\text { Physiological trigger for } \\
\text { fluid administration }\end{array}$ & $\begin{array}{l}\text { Physiological } \\
\text { end-point for fluid } \\
\text { administration }\end{array}$ & $\begin{array}{l}\text { Number of } \\
\text { boluses } \\
\text { administered }\end{array}$ & $\begin{array}{l}\text { Vasoactive } \\
\text { administration? }\end{array}$ & $\begin{array}{l}\text { Packed red } \\
\text { cell transfusion? }\end{array}$ \\
\hline \multirow[t]{6}{*}{ Bihari [36] } & \multirow[t]{6}{*}{2013} & \multirow[t]{6}{*}{ Undefined } & $4 \%$ albumin & 750 & \multirow[t]{6}{*}{$<30$} & \multirow[t]{6}{*}{ Clinician defined } & \multirow[t]{6}{*}{ Clinician defined } & \multirow[t]{6}{*}{2} & \multirow[t]{6}{*}{ Yes } & \multirow[t]{6}{*}{ Not described } \\
\hline & & & $\begin{array}{l}\text { Packed } \\
\text { red cells }\end{array}$ & & & & & & & \\
\hline & & & $20 \%$ albumin & & & & & & & \\
\hline & & & $\begin{array}{l}\text { Fresh frozen } \\
\text { plasma }\end{array}$ & & & & & & & \\
\hline & & & $4 \%$ gelatin & & & & & & & \\
\hline & & & $0.9 \%$ saline & & & & & & & \\
\hline \multirow{2}{*}{$\begin{array}{l}\text { Castellanos- } \\
\text { Ortega [37] }\end{array}$} & \multirow[t]{2}{*}{2010} & \multirow[t]{2}{*}{ Undefined } & Crystalloid & 1,000 & \multirow[t]{2}{*}{30} & \multirow[t]{2}{*}{ Hypotension } & \multirow{2}{*}{$\begin{array}{l}\mathrm{CVP} \geq 8 \mathrm{mmHg} \\
\mathrm{MAP} \geq 65 \mathrm{mmHg} \\
\mathrm{ScVO}_{2} \geq 70 \%\end{array}$} & \multirow[t]{2}{*}{ Not described } & \multirow[t]{2}{*}{ Yes } & \multirow[t]{2}{*}{ Not described } \\
\hline & & & Colloid & 500 & & & & & & \\
\hline \multirow[t]{2}{*}{ De Backer [38] } & \multirow[t]{2}{*}{2010} & \multirow{2}{*}{$\begin{array}{l}500 \mathrm{ml} \text { colloid or } \\
1,000 \mathrm{ml} \text { crystalloid }\end{array}$} & Crystalloid & 1,000 & \multirow[t]{2}{*}{ Not defined } & \multirow{2}{*}{$\begin{array}{l}\text { MAP }<70 \mathrm{mmHg} \text {; SBP } \\
<100 \mathrm{mmHg} \text {, altered } \\
\text { mental state; mottled } \\
\text { skin; oliguria }>1 \text { hour, } \\
\text { hyperlactataemia }\end{array}$} & \multirow[t]{2}{*}{ Not described } & \multirow[t]{2}{*}{ Not described } & \multirow[t]{2}{*}{ Yes } & \multirow[t]{2}{*}{ Not described } \\
\hline & & & Colloid & 500 & & & & & & \\
\hline Dong [39] & 2012 & Undefined & $6 \% \mathrm{HES}$ & 500 & 30 & $\begin{array}{l}\text { SBP }<90 \mathrm{mmHg} \text { or } \\
>40 \mathrm{mmHg} \text { drop } \\
\text { or need for } \\
\text { vasopressors, } \\
\text { oliguria >1 hour; } \\
\text { mottled skin; HR } \\
>100 \text { bpm }\end{array}$ & End of infusion. & 1 & Not described & Not described \\
\hline Freitas [40] & 2012 & Undefined & $6 \%$ HES & $\begin{array}{l}7 \mathrm{ml} / \mathrm{kg} \\
(\max 500)\end{array}$ & 30 & Clinician defined & End of infusion & 1 & Yes & No \\
\hline Gaieski [41] & 2010 & $20-30 \mathrm{ml} / \mathrm{kg}$ & $0.9 \%$ saline & 500 & $15-20$ & CVP $<8 \mathrm{mmHg}$ & CVP $>8 \mathrm{mmHg}$ & Not described & Yes & Yes \\
\hline Hamzaoui [42] & 2010 & Undefined & $0.9 \%$ saline & 1,000 & Not defined & Undefined & Not described & Not described & Yes & Not described \\
\hline \multirow[t]{2}{*}{ Hanzelka [43] } & \multirow[t]{2}{*}{2013} & \multirow[t]{2}{*}{$20 \mathrm{ml} / \mathrm{kg}$} & \multirow[t]{2}{*}{ Undefined } & 1,000 & 60 & \multirow[t]{2}{*}{ Severe sepsis } & \multirow{2}{*}{$\begin{array}{l}\text { SBP }>90 \mathrm{mmHg} \\
\text { MAP }<65 \mathrm{mmHg}\end{array}$} & Not described & Yes & No \\
\hline & & & & 500 & 30 & & & & & \\
\hline Jacob [44] & 2012 & Undefined & $0.9 \%$ saline & 1,000 & 60 & $\mathrm{SBP}<100 \mathrm{mmHg}$ & SBP increased by & Up to 10 & No & Not described \\
\hline & & & & 500 & 30 & or hyperlactataemia & $\begin{array}{l}10 \mathrm{mmHg} \text { for } 2 \\
\text { consecutive hours } \\
\text { to }>90 \mathrm{mmHg}\end{array}$ & & & \\
\hline Khwannimit [45] & 2012 & Undefined & $6 \%$ HES & 500 & 30 & Clinician defined & End of infusion & 1 & Yes & Not described \\
\hline Lakhal [46] & 2013 & Undefined & $4 \%$ gelatin & 500 & 30 & $\begin{array}{l}\text { One or more of SBP } \\
<90 \mathrm{mmHg} \text {, MAP } \\
<65 \mathrm{mmHg} \text {, requiring } \\
\text { vasoactive medication, } \\
\text { oliguria, skin mottling, } \\
\text { hyperlactataemia }\end{array}$ & End of infusion & 1 & Yes & Not described \\
\hline
\end{tabular}


Table 2 Description of fluid boluses, triggers, physiological end-points and primary confounders (Continued)

\begin{tabular}{|c|c|c|c|c|c|c|c|c|c|c|c|}
\hline Lanspa [47] & 2012 & $5,060 \mathrm{ml}$ & $\begin{array}{l}\text { Crystalloid } \\
\text { (or equivalent } \\
\text { colloid) }\end{array}$ & 20 ml/kg & & $<20$ & Clinician defined & End of infusion & 1.36 & Yes & Yes \\
\hline $\begin{array}{l}\text { Machare-Delgado } \\
\text { [48] }\end{array}$ & 2011 & Undefined & $0.9 \%$ saline & 500 & 10 & & Clinician defined & End of infusion & 1 & Not described & No \\
\hline $\begin{array}{l}\text { MacRedmond } \\
\text { [49] }\end{array}$ & 2010 & $25 \mathrm{ml} / \mathrm{kg}$ & $0.9 \%$ saline & 500 & & $<15$ & MAP $<65 \mathrm{mmHg}$ & $\begin{array}{l}\text { CVP 8-12; MAP } \\
>65 \mathrm{mmHg}^{2} \\
\mathrm{ScVO}_{2}>70 \%\end{array}$ & Not described & Yes & Yes \\
\hline Mahjoub [50] & 2013 & Undefined & $0.9 \%$ saline & 500 & 20 & & $\begin{array}{l}\text { SBP }<90 \mathrm{mmHg} \text { and/or } \\
\text { need for vasoactive } \\
\text { drugs and/or persistent } \\
\text { lactic acidosis }\end{array}$ & End of infusion & 1 & Yes & Not described \\
\hline McIntyre [51] & 2012 & $2,400 \mathrm{ml}$ & $\begin{array}{l}0.9 \% \text { saline or } \\
4 \% \text { albumin }\end{array}$ & 500 & & STAT & Undefined & Not described & 6 & Yes & Not described \\
\hline Monnet [52] & 2010 & Undefined & $0.9 \%$ saline & 500 & 30 & & $\begin{array}{l}\text { SBP }<90 \mathrm{mmHg} \text {, SBP } \\
\text { drop }>50 \mathrm{mmHg} \text { if } \mathrm{HT} \text {, } \\
\text { and one or more of } \\
\mathrm{HR}>100 \text {, skin mottling } \\
\text { or oliguria }\end{array}$ & End of infusion & 1 & Yes & Not described \\
\hline Monnet [53] & 2011 & $2,200 \mathrm{ml}$ & $0.9 \%$ saline & 500 & 10 & & $\begin{array}{l}\text { SBP }<90 \mathrm{mmHg} \text {, SBP } \\
\text { drop }>50 \mathrm{mmHg} \text { if HT, } \\
\text { and one or more of HR } \\
>100 \text {, skin mottling or } \\
\text { oliguria }\end{array}$ & End of infusion & 1 & Yes & Not described \\
\hline Monnet [54] & 2013 & Undefined & 0.9\% saline & 500 & 30 & & $\begin{array}{l}\text { SBP }<90 \mathrm{mmHg} \text {, SBP } \\
\text { drop }>50 \mathrm{mmHg} \text { if HT, } \\
\text { and one or more of HR } \\
>100 \text {, skin mottling or } \\
\text { oliguria }\end{array}$ & End of infusion & 1 & Yes & Yes \\
\hline Monnet [55] & 2011 & Undefined & $0.9 \%$ saline & 500 & 20 & & $\begin{array}{l}\text { SBP }<90 \mathrm{mmHg} \text {, SBP } \\
\text { drop }>50 \mathrm{mmHg} \text { if HT, } \\
\text { and one or more of } \\
\mathrm{HR}>100 \text {, skin mottling } \\
\text { or oliguria }\end{array}$ & End of infusion & 1 & Yes & Not described \\
\hline O Neill [56] & 2012 & $20 \mathrm{ml} / \mathrm{kg}$ & $0.9 \%$ saline & 500 & 15 & & $\begin{array}{l}\text { CVP }<8 \text { mmHg; } \\
\text { MAP <65 mmHg; } \\
\text { SCVO }_{2}<70 \%\end{array}$ & $\begin{array}{l}\text { CVP 8-12; MAP } \\
>65 \mathrm{mmHg}^{2} \\
\mathrm{ScVO}_{2}>70 \%\end{array}$ & 0.68 & Yes & Not described \\
\hline \multirow{2}{*}{$\begin{array}{l}\text { Ospina-Tascon } \\
\text { [57] }\end{array}$} & \multirow[t]{2}{*}{2010} & \multirow[t]{2}{*}{ Undefined } & CSL & 1,000 & \multirow{2}{*}{\multicolumn{2}{|c|}{30}} & \multirow[t]{2}{*}{ MAP $<65 \mathrm{mmHg}$} & \multirow[t]{2}{*}{ End of infusion } & \multirow[t]{2}{*}{1} & \multirow[t]{2}{*}{ Yes } & \multirow[t]{2}{*}{ Not describec } \\
\hline & & & $4 \%$ albumin & 400 & & & & & & & \\
\hline Patel [58] & 2010 & $2,000 \mathrm{ml}$ & Normal saline & Undefined & 30 & & $\begin{array}{l}\text { SBP }<90 \mathrm{mmHg} \\
\text { MAP }<65 \mathrm{mmHg}\end{array}$ & Not described & 1 & Yes & Not described \\
\hline Pierrakos [59] & 2012 & Undefined & CSL & 100 & 30 & & Clinician defined & End of infusion & 1 & Yes & Not described \\
\hline
\end{tabular}


Table 2 Description of fluid boluses, triggers, physiological end-points and primary confounders (Continued)

\begin{tabular}{|c|c|c|c|c|c|c|c|c|c|c|}
\hline Pottecher [60] & 2010 & Undefined & $\begin{array}{l}\text { HES } 6 \% \text { or } \\
0.9 \% \text { saline }\end{array}$ & 500 & 30 & $\begin{array}{l}\text { MAP }<65 \text { mmHg, skin } \\
\text { mottling or oliguria }\end{array}$ & End of infusion & 1 & Yes & Not described \\
\hline \multirow[t]{2}{*}{ Sanchez [61] } & \multirow[t]{2}{*}{2011} & \multirow[t]{2}{*}{ Undefined } & Crystalloid & 1,000 & \multirow[t]{2}{*}{ Undefined } & \multirow{2}{*}{$\begin{array}{l}\text { Hypotension with } \\
\text { perfusion abnormalities }\end{array}$} & & \multirow[t]{2}{*}{ Not described } & \multirow[t]{2}{*}{ Yes } & \multirow[t]{2}{*}{ No } \\
\hline & & & Colloid & 500 & & & $\begin{array}{l}\text { ITBVI }>900 \mathrm{ml} / \mathrm{ml} \\
\text { or EVLWI }>10 \mathrm{ml} / \mathrm{kg}\end{array}$ & & & \\
\hline Schnell [62] & 2013 & Undefined & $0.9 \%$ saline & 500 & $15-30$ & Clinician defined & End of infusion & 1 & Yes & Not described \\
\hline Sturgess [63] & 2010 & Undefined & $4 \%$ albumin & 250 & 15 & Clinician defined & End of infusion & 1 & Yes & No \\
\hline Trof [64] & 2012 & Undefined & $\begin{array}{l}\text { HES or } 4 \% \\
\text { gelatin }\end{array}$ & $250-500$ & 30 & $\begin{array}{l}\text { EVLWI }<10 \mathrm{ml} / \mathrm{kg} \text { or } \\
>10 \mathrm{ml} / \mathrm{kg} \text { with GEDVI } \\
<850 \mathrm{ml} / \mathrm{m}^{2} ; \mathrm{PAOP} \\
>18 \mathrm{mmHg} \text { MAP } \\
<65 \mathrm{mmH} \text {, HR } \\
>100, \mathrm{SVO}_{2}<65 \% \\
\text { or } \mathrm{ScVO}_{2}<70 \% \text {; } \\
\text { oliguria; peripheral } \\
\text { perfusion deficits, } \\
\text { hyperlactatemia }\end{array}$ & $\begin{array}{l}\text { MAP }>65 \mathrm{mmHg} \text {, } \\
\mathrm{ScvO}_{2}>70 \% \text {, lactate } \\
\text { clearance, diuresis } \\
>0.5 \mathrm{ml} / \mathrm{kg} / \text { hour, } \\
\text { restoration of } \\
\text { peripheral } \\
\text { perfusion deficits }\end{array}$ & 3.48 & Yes & Not described \\
\hline \multirow[t]{3}{*}{ van Haren [65] } & \multirow[t]{3}{*}{2012} & \multirow[t]{3}{*}{ Undefined } & \multirow{2}{*}{$\begin{array}{l}6 \% \text { HES in } \\
0.9 \% \text { saline }\end{array}$} & \multirow{3}{*}{$\begin{array}{l}500 \\
250\end{array}$} & \multirow{3}{*}{$\begin{array}{l}15 \\
15\end{array}$} & \multirow[t]{3}{*}{ Septic shock } & \multirow[t]{3}{*}{ End of infusion } & \multirow[t]{3}{*}{1} & \multirow[t]{3}{*}{ Yes } & \multirow[t]{3}{*}{ Not described } \\
\hline & & & & & & & & & & \\
\hline & & & $\begin{array}{l}6 \% \text { HES in } \\
7.2 \% \text { saline }\end{array}$ & & & & & & & \\
\hline Wacharasint [66] & 2013 & Undefined & HES $6 \%$ & 500 & 30 & $\begin{array}{l}\mathrm{SBP}<90 \mathrm{mmHg} \text { or } \\
\text { requirement for } \\
\text { vasopressors }\end{array}$ & End of infusion & 1 & Yes & Not described \\
\hline \multirow[t]{2}{*}{ Yu [67] } & \multirow[t]{2}{*}{2011} & \multirow{2}{*}{$\begin{array}{l}30 \mathrm{ml} / \mathrm{kg} \\
\text { in } 1,000 \mathrm{ml} \\
\text { increments }\end{array}$} & \multirow[t]{2}{*}{$\begin{array}{l}\text { Crystalloid } \\
\text { or colloid }\end{array}$} & \multirow[t]{2}{*}{$250-500$} & \multirow[t]{2}{*}{ Undefined } & $\begin{array}{l}\text { PAOP }<12 \mathrm{mmHg} \text { or } \\
12-17 \mathrm{mmHg} \text { with }\end{array}$ & \multirow{2}{*}{$\begin{array}{l}\mathrm{SBP}>100 \mathrm{mmHg} \\
\mathrm{HR}<100 \mathrm{bpm} \\
\mathrm{UO}>0.5 \mathrm{ml} / \mathrm{kg} / \mathrm{hour}, \\
\text { lactate clearance, } \\
\mathrm{SmvO}_{2}>70 \%\end{array}$} & \multirow[t]{2}{*}{ Not described } & \multirow[t]{2}{*}{ Not described } & \multirow[t]{2}{*}{ Yes } \\
\hline & & & & & & $\begin{array}{l}\mathrm{SBP}<100 ; \\
\mathrm{HR}>100 \mathrm{bpm} \\
\mathrm{UO}<0.5 \mathrm{ml} / \mathrm{kg} / \text { hour; } \\
\text { hyperlactataemia; } \mathrm{SVO}_{2} \\
>70 \% \text { or equivalent } \\
\text { blood volume goals }\end{array}$ & & & & \\
\hline Zhang [68] & 2012 & Undefined & $\begin{array}{l}\text { Crystalloid } \\
\text { or colloid }\end{array}$ & $250-500$ & 30 & $\begin{array}{l}\text { SBP }<90 \mathrm{mmHg} ; \mathrm{HR} \\
>100 \mathrm{bpm} ; \mathrm{GEDVI} \\
<700 \mathrm{ml} / \mathrm{m}^{2} ; \text { CVP } \\
<12 \mathrm{mmHg} \text { (PEEP } \\
\text { dependent) }\end{array}$ & $\begin{array}{l}\text { Pre-defined } \\
\text { rise in CVP }\end{array}$ & Not described & Yes & Not described \\
\hline
\end{tabular}

CSL, compound sodium lactate solution; CVP, central venous pressure; EVLWI, extra-vascular lung water index; HES, hydroxyethyl starch; HR, heart rate; $\mathrm{HT}$, hypertensive; GEDVI, global end diastolic volume index; ITBVI,

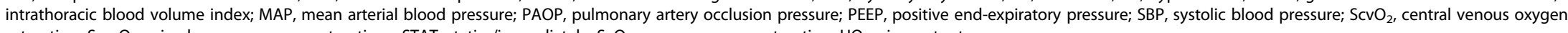
saturation; $\mathrm{SmVO}_{2}$, mixed venous oxygen saturations; STAT, statim/immediately; $\mathrm{SVO}_{2}$, venous oxygen saturation; $\mathrm{UO}$, urine output. 
Temporal trends in physiological changes following fluid bolus therapy

The temporal change in physiological parameters following FBT is described in 31 different groups across 17 studies (Table 3 ).

\section{Immediately post-infusion}

Ten studies reported the physiological state after bolus administration in 18 groups immediately post-administration. In the six studies describing changes in cardiac index immediately post-FBT, cardiac index increased by a median of $800 \mathrm{ml} / \mathrm{minute} / \mathrm{m}^{2}$ (range 0 to $1,300 \mathrm{ml} / \mathrm{mi}$ nute $/ \mathrm{m}^{2}$ ). The median reduction in heart rate at the end of a fluid bolus (eight studies) was $2 \mathrm{bpm}$ (range 10 to $0 \mathrm{bpm}$ reduction) and the median increase in mean arterial pressure (eight studies) was $7 \mathrm{mmHg}$ (range 1 to $15.2 \mathrm{mmHg}$ ). The median increase in CVP across five studies was $3.2 \mathrm{mmHg}$ (range 2.3 to $5.2 \mathrm{mmHg}$ ). Only a single study reported the effect on venous oxygen saturation, blood lactate concentration or haemoglobin concentration. No study reported the effect on urine output.

\section{Thirty minutes post-administration}

Five studies reported the physiological effects of FBT 30 minutes after administration. Cardiac index increased by a median of $300 \mathrm{ml} / \mathrm{minute} / \mathrm{m}^{2}$ (range -400 to $600 \mathrm{ml} / \mathrm{minute} / \mathrm{m}^{2}$ ) in three studies. The median reduction in heart rate (five studies) was $2 \mathrm{bpm}$ (range $11 \mathrm{bpm}$ reduction to $0.3 \mathrm{bpm}$ increase) and the median increase in mean arterial pressure (five studies) was $7.5 \mathrm{mmHg}$ (range 3 to $11 \mathrm{mmHg}$ ). The median increase in CVP across four studies was $3 \mathrm{mmHg}$ (range 2 to $5.25 \mathrm{mmHg}$ ). There was a median increase in central venous saturation of $2 \%$ (range $4 \%$ reduction to $8 \%$ increase) across two studies. Changes in other indices are reported in Table 3.

\section{Sixty minutes post-administration}

Only three studies reported the physiological effects of FBT 60 minutes after administration (Figure 4) $[36,57,65]$. Cardiac index increased by a median of $300 \mathrm{ml} / \mathrm{minute} / \mathrm{m}^{2}$ (range -300 to $400 \mathrm{ml} / \mathrm{minute} / \mathrm{m}^{2}$ ) in two studies. The median reduction in heart rate $60 \mathrm{mi}-$ nutes after a fluid bolus (three studies) was $1 \mathrm{bpm}$ (range $11 \mathrm{bpm}$ reduction to $2 \mathrm{bpm}$ increase) and the median increase in mean arterial pressure (three studies) was $3 \mathrm{mmHg}$ (range 2 to $7 \mathrm{mmHg}$ ). The median increase in CVP across three studies was $2 \mathrm{mmHg}$ (range 1 to $3 \mathrm{mmHg}$ ). There was a median increase in central venous saturation of $1 \%$ (range $0.4 \%$ to $2 \%$ increase) across two studies.

\section{Beyond 1 hour post-fluid bolus therapy}

Only one study reported the effects of BFT at 120, 180 and 240 minutes after administration (Figure 4) [65].

\section{Comparing responders and non-responders}

Overall, 10 studies compared the physiological responses to FBT administration between groups defined by changes in a physiological variable. Patients were defined as either responders or non-responders depending on the response exhibited. Different variables are used in different studies: stroke volume index (five studies), cardiac index or output (three studies), increase in oxygen consumption (one study) or aortic blood flow rate (one study). All reported changes only within 30 minutes of FBT completion (Additional file 1: Table S2).

In the six studies describing changes in cardiac index, cardiac index increased by a median of $850 \mathrm{ml} / \mathrm{minute} /$ $\mathrm{m}^{2}$ (range 600 to $1,300 \mathrm{ml} / \mathrm{minute} / \mathrm{m}^{2}$ ) in fluid responders compared with $200 \mathrm{ml} /$ minute $/ \mathrm{m}^{2}$ (range 0 to $1,000 \mathrm{ml} / \mathrm{minute} / \mathrm{m}^{2}$ ) in non-responders. The median increase in mean arterial pressure (10 studies) in responders was $9.5 \mathrm{mmHg}$ (range 7 to $15.2 \mathrm{mmHg}$ ) versus $4.8 \mathrm{mmHg}$ (range 1 to $13 \mathrm{mmHg}$ ) in non-responders. Similarly, the median increase in central venous pressure (six studies) was $3 \mathrm{mmHg}$ (range 2.6 to $3.4 \mathrm{mmHg}$ ) in responders versus $3.7 \mathrm{mmHg}$ (range 2 to $5.2 \mathrm{mmHg}$ ) in non-responders. The median decrease in heart rate (nine studies) was $3.3 \mathrm{bpm}$ in responders (range 1.5 to $10 \mathrm{bpm}$ decrease) and $1.2 \mathrm{bpm}$ in non-responders (range 0 to $4 \mathrm{bpm}$ decrease). Information on changes in venous oxygen saturation, blood lactate concentration, and blood haemoglobin concentration in the few studies reporting such data are presented in Additional file 1: Table S2.

\section{Additional comparisons}

The physiological effects of FBT grouped by speed of FBT delivery (Additional file 1: Table S3) and by class of fluid administered (Additional file 1: Table S4) have also been presented. There is no consistent pattern demonstrated across or between groups.

\section{Relationship between physiological changes after fluid bolus therapy and clinical outcome}

Overall, seven studies described clinically orientated outcomes $[37,43,44,49,58,59,64]$. All reported the effects of complex interventions, such as early goal-directed therapy. No studies examined the relationship between FBT and outcome directly (Tables 4 and 5).

\section{Discussion}

We examined the contemporary literature on FBT in severe sepsis and septic shock and identified 33 original studies describing the characteristics of a fluid bolus, 17 of which also describe the associated physiological 
Table 3 Physiological effects grouped by measurement time

\begin{tabular}{|c|c|c|c|c|c|c|c|c|}
\hline First author & Fluid given & Group & $\begin{array}{l}\text { Time from completion } \\
\text { of fluid administration } \\
\text { until physiological } \\
\text { measurement (minutes) }\end{array}$ & $\begin{array}{l}\text { Measure } \\
\text { of central } \\
\text { tendency }\end{array}$ & $\begin{array}{l}\text { Change in } \\
\text { cardiac output } \\
\text { estimation }\end{array}$ & $\begin{array}{l}\text { Change in } \\
\text { heart rate } \\
\text { (bpm) }\end{array}$ & $\begin{array}{l}\text { Change } \\
\text { in mean } \\
\text { arterial } \\
\text { pressure } \\
(\mathrm{mmHg}))\end{array}$ & $\begin{array}{l}\text { Chang } \\
\text { in cent } \\
\text { venous } \\
\text { pressu } \\
\text { (mmHc }\end{array}$ \\
\hline \multicolumn{9}{|c|}{ Haemodynamic indices measured immediately following fluid bolus administration } \\
\hline \multirow[t]{2}{*}{$\begin{array}{l}\text { Machare- } \\
\text { Delgado [48] }\end{array}$} & $\begin{array}{l}500 \mathrm{ml} \text { of } 0.9 \% \\
\text { saline over } \\
10 \text { minutes }\end{array}$ & $\begin{array}{l}\text { Responders: } \\
>10 \% \mathrm{SVl} \\
\text { increase }\end{array}$ & 0 & Mean & $\begin{array}{l}+3.99 \mathrm{ml} / \\
\mathrm{m}^{2} / \text { beat }\end{array}$ & & & \\
\hline & $\begin{array}{l}500 \mathrm{ml} \text { of } 0.9 \% \\
\text { saline over } \\
10 \text { minutes }\end{array}$ & $\begin{array}{l}\text { Non-responders: } \\
>10 \% \mathrm{SVl} \\
\text { increase }\end{array}$ & 0 & Mean & $\begin{array}{l}+0.57 \mathrm{ml} / \\
\mathrm{m}^{2} / \text { beat }\end{array}$ & & & \\
\hline \multirow[t]{2}{*}{ Dong [39] } & $\begin{array}{l}500 \mathrm{ml} \text { of } 6 \% \\
\text { HES over } \\
30 \text { minutes }\end{array}$ & $\begin{array}{l}\text { Responders: } \\
>15 \% \mathrm{SVI} \\
\text { increase }\end{array}$ & 0 & Mean & $\begin{array}{l}+600 \mathrm{ml} / \\
\mathrm{min} / \mathrm{m}^{2}\end{array}$ & -1.5 & +15.2 & +3.2 \\
\hline & $\begin{array}{l}500 \mathrm{ml} \text { of } 6 \% \\
\text { HES over } \\
30 \text { minutes }\end{array}$ & $\begin{array}{l}\text { Non-responders: } \\
<15 \% \mathrm{SVl} \\
\text { increase }\end{array}$ & 0 & Mean & $\begin{array}{l}+300 \mathrm{ml} / \\
\mathrm{min} / \mathrm{m}^{2}\end{array}$ & -1.2 & +4.8 & +2.3 \\
\hline \multirow[t]{2}{*}{$\begin{array}{l}\text { Khwannimit } \\
\text { [45] }\end{array}$} & $\begin{array}{l}500 \mathrm{ml} \text { of } 6 \% \\
\text { HES over } \\
30 \text { minutes }\end{array}$ & $\begin{array}{l}\text { Responders: } \\
>15 \% \mathrm{SVI} \\
\text { increase }\end{array}$ & 0 & Mean & $\begin{array}{l}+1300 \mathrm{ml} / \\
\mathrm{min} / \mathrm{m}^{2}\end{array}$ & -3.3 & +9.5 & +3.4 \\
\hline & $\begin{array}{l}500 \mathrm{ml} \text { of } \\
6 \% \text { HES over } \\
30 \text { minutes }\end{array}$ & $\begin{array}{l}\text { Non-responders: } \\
<15 \% \mathrm{SVl} \\
\text { increase }\end{array}$ & 0 & Mean & $\begin{array}{l}+200 \mathrm{ml} / \\
\mathrm{min} / \mathrm{m}^{2}\end{array}$ & -0.9 & +3.9 & +5.2 \\
\hline \multirow[t]{2}{*}{ Lakhal [46] } & $\begin{array}{l}500 \mathrm{ml} \text { of } 4 \% \\
\text { gelatin over } \\
30 \text { minutes }\end{array}$ & $\begin{array}{l}\text { Responders: } \\
>15 \% \mathrm{SVI} \\
\text { increase }\end{array}$ & 0 & Mean & $\begin{array}{l}+900 \mathrm{ml} / \\
\mathrm{min} / \mathrm{m}^{2}\end{array}$ & -6 & +14 & +3 \\
\hline & $\begin{array}{l}500 \mathrm{ml} \text { of } 4 \% \\
\text { gelatin over } \\
30 \text { minutes }\end{array}$ & $\begin{array}{l}\text { Non-responders: } \\
<15 \% \mathrm{SVl} \\
\text { increase }\end{array}$ & 0 & Mean & $\begin{array}{l}+0 \mathrm{ml} / \\
\mathrm{min} / \mathrm{m}^{2}\end{array}$ & -3 & +7 & +4.5 \\
\hline \multirow[t]{2}{*}{ Mahjoub [50] } & $\begin{array}{l}500 \mathrm{ml} \text { of } 0.9 \% \\
\text { saline over } \\
20 \text { minutes }\end{array}$ & $\begin{array}{l}\text { Responders: } \\
>10 \% \mathrm{SV} \\
\text { increase }\end{array}$ & 0 & Mean & $+1,000 \mathrm{ml} / \mathrm{min}$ & -4 & +7 & +2.6 \\
\hline & $\begin{array}{l}500 \mathrm{ml} \text { of } 0.9 \% \\
\text { saline over } \\
20 \text { minutes }\end{array}$ & $\begin{array}{l}\text { Non-responders: } \\
>10 \% \mathrm{SV} \\
\text { increase }\end{array}$ & 0 & Mean & $+300 \mathrm{ml} / \mathrm{min}$ & -3 & +1 & +2.9 \\
\hline Monnet [53] & $\begin{array}{l}500 \mathrm{ml} \text { of } 0.9 \% \\
\text { saline over } \\
10 \text { minutes }\end{array}$ & All patients & 0 & Mean & $\begin{array}{l}+800 \mathrm{ml} / \\
\mathrm{min} / \mathrm{m}^{2}\end{array}$ & -7 & +8 & +5 \\
\hline
\end{tabular}


Table 3 Physiological effects grouped by measurement time (Continued)

\begin{tabular}{|c|c|c|c|c|c|c|c|c|c|c|c|}
\hline \multirow[t]{2}{*}{ Monnet [55] } & $\begin{array}{l}500 \mathrm{ml} \text { of } 0.9 \% \\
\text { saline over } \\
20 \text { minutes }\end{array}$ & $\begin{array}{l}\text { Responders: } \\
>15 \% \mathrm{Cl} \\
\text { increase }\end{array}$ & 0 & Mean & $\begin{array}{l}+800 \mathrm{ml} / \\
\mathrm{min} / \mathrm{m}^{2}\end{array}$ & -2 & +11 & & & & \\
\hline & $\begin{array}{l}500 \mathrm{ml} \text { of } 0.9 \% \\
\text { saline over } \\
20 \text { minutes }\end{array}$ & $\begin{array}{l}\text { Non-responders: } \\
<15 \% \text { increase } \\
\text { in Cl }\end{array}$ & 0 & Mean & $\begin{array}{l}+200 \mathrm{ml} / \\
\mathrm{min} / \mathrm{m}^{2}\end{array}$ & -2 & +4 & & & & \\
\hline \multirow[t]{2}{*}{ Monnet [54] } & $\begin{array}{l}500 \mathrm{ml} \text { of } 0.9 \% \\
\text { saline over } \\
30 \text { minutes }\end{array}$ & $\begin{array}{l}\text { Responders: } \\
>15 \% \mathrm{VO}_{2} \\
\text { increase }\end{array}$ & 0 & Mean & $\begin{array}{l}+1,000 \mathrm{ml} / \\
\mathrm{min} / \mathrm{m}^{2}\end{array}$ & -2 & +7 & & $+1 \%$ & -1.9 & -7 \\
\hline & $\begin{array}{l}500 \mathrm{ml} \text { of } 0.9 \% \\
\text { saline over } \\
30 \text { minutes }\end{array}$ & $\begin{array}{l}\text { Non-responders: } \\
<15 \% \text { increase } \\
\text { in } \mathrm{VO}_{2}\end{array}$ & 0 & Mean & $\begin{array}{l}+1,000 \mathrm{ml} / \\
\mathrm{min} / \mathrm{m}^{2}\end{array}$ & +0 & +13 & & $+7 \%$ & -0.3 & -6 \\
\hline \multirow[t]{2}{*}{ Schnell [62] } & $\begin{array}{l}500 \mathrm{ml} \text { of } 0.9 \% \\
\text { saline over } \\
15-30 \text { minutes }\end{array}$ & $\begin{array}{l}\text { Responders: } \\
>10 \% \text { increase } \\
\text { in aortic blood } \\
\text { flow }\end{array}$ & 0 & Median & $+20 \mathrm{ml} /$ beat & -10 & +7 & & & & \\
\hline & $\begin{array}{l}500 \mathrm{ml} \text { of } 0.9 \% \\
\text { saline over } \\
15-30 \text { minutes }\end{array}$ & $\begin{array}{l}\text { Non-responders: } \\
<10 \% \text { increase in } \\
\text { aortic blood flow }\end{array}$ & 0 & Median & $+8 \mathrm{ml} /$ beat & -1 & +6 & & & & \\
\hline Sturgess [63] & $\begin{array}{l}250 \mathrm{ml} \text { of } 4 \% \\
\text { albumin over } \\
15 \text { minutes }\end{array}$ & All patients & 0 & Mean & $\begin{array}{l}+7.5 \% \\
\mathrm{ml} / \text { beat }\end{array}$ & & & & & & \\
\hline \multicolumn{12}{|c|}{ Haemodynamic indices measured 30 minutes after fluid bolus administration } \\
\hline \multirow[t]{2}{*}{ Freitas [40] } & $\begin{array}{l}7 \mathrm{ml} / \mathrm{kg} \text {, } \\
\text { maximum } \\
500 \mathrm{ml} \text {, of } 6 \% \\
\text { HES over } \\
30 \text { minutes }\end{array}$ & $\begin{array}{l}\text { Responders: } \\
\text { >15\% CO } \\
\text { increase }\end{array}$ & 30 & Mean & $+2,100 \mathrm{ml} / \mathrm{min}$ & -2 & +11 & +3 & $+8 \%$ & -0.1 & \\
\hline & $\begin{array}{l}7 \mathrm{ml} / \mathrm{kg} \text {, } \\
\text { maximum } \\
500 \mathrm{ml} \text {, of } 6 \% \\
\text { HES over } \\
30 \text { minutes }\end{array}$ & $\begin{array}{l}\text { Non-responders: } \\
<15 \% \text { increase } \\
\text { in CO }\end{array}$ & 30 & Mean & $+200 \mathrm{ml} / \mathrm{min}$ & +0 & +8 & +5 & $-3.5 \%$ & -0.2 & \\
\hline \multirow[t]{2}{*}{ Pierrakos [59] } & $\begin{array}{l}500 \mathrm{ml} \text { of } 6 \% \\
\text { HES or } 1,000 \mathrm{ml} \\
\text { of CSL over } \\
30 \text { minutes }\end{array}$ & $\begin{array}{l}\text { Responders: } \\
>10 \% \text { increase } \\
\text { in Cl }\end{array}$ & 30 & Mean & $\begin{array}{l}+600 \mathrm{ml} / \\
\mathrm{min} / \mathrm{m}^{2}\end{array}$ & -4 & +8 & +3 & $+3 \%$ & & \\
\hline & $\begin{array}{l}500 \mathrm{ml} \text { of } 6 \% \\
\text { HES or } 1,000 \mathrm{ml} \\
\text { of CSL over } \\
30 \text { minutes }\end{array}$ & $\begin{array}{l}\text { Non-responders: } \\
<10 \% \text { increase } \\
\text { in } \mathrm{Cl}\end{array}$ & 30 & Mean & $\begin{array}{l}+0 \mathrm{ml} / \\
\mathrm{min} / \mathrm{m}^{2}\end{array}$ & -4 & +3 & +2 & $+0 \%$ & & \\
\hline Pottecher [60] & $\begin{array}{l}\text { Up to } 500 \mathrm{ml} \\
\text { of } 6 \% \mathrm{HES} \text { or } \\
0.9 \% \text { saline over } \\
30 \text { minutes }\end{array}$ & All patients & 30 & Mean & $+1,400 \mathrm{ml} / \mathrm{min}$ & -2 & +7 & & & & \\
\hline
\end{tabular}


Table 3 Physiological effects grouped by measurement time (Continued)

\begin{tabular}{|c|c|c|c|c|c|c|c|c|c|c|c|c|}
\hline $\begin{array}{l}\text { Wacharasint } \\
\text { [66] }\end{array}$ & $\begin{array}{l}500 \mathrm{ml} \text { of } 6 \% \\
\text { HES over } \\
30 \text { minutes }\end{array}$ & All patients & 30 & Mean & $\begin{array}{l}+470 \mathrm{ml} / \\
\mathrm{min} / \mathrm{m}^{2}\end{array}$ & +0.3 & +9.2 & +5.25 & & & & \\
\hline \multirow[t]{2}{*}{$\begin{array}{l}\text { van Haren } \\
\text { [65] }\end{array}$} & $\begin{array}{l}250 \mathrm{ml} \text { of } 6 \% \\
\text { HES in } 7.2 \% \\
\text { saline over } \\
15 \text { minutes }\end{array}$ & Hypertonic bolus & 30 & Mean & $\begin{array}{l}+300 \mathrm{ml} / \\
\mathrm{min} / \mathrm{m}^{2}\end{array}$ & -11 & +4 & +2 & & -0.2 & & -8 \\
\hline & $\begin{array}{l}500 \mathrm{ml} \text { of } 6 \% \\
\text { HES in } 0.9 \% \\
\text { saline over } \\
15 \text { minutes }\end{array}$ & Isotonic bolus & 30 & Mean & $\begin{array}{l}-400 \mathrm{ml} / \\
\mathrm{min} / \mathrm{m}^{2}\end{array}$ & -1 & +5 & +4 & & -0.1 & & -9 \\
\hline \multicolumn{13}{|c|}{ Haemodynamic indices measured 60 minutes after fluid bolus administration } \\
\hline Bihari [36] & $\begin{array}{l}500-750 \mathrm{ml} \text { of } \\
4 \% \text { albumin, } \\
\text { blood, 20\% } \\
\text { albumin FFP, } \\
0.9 \% \text { saline, } 4 \% \\
\text { gelatin or } \\
\text { platelets } \\
\text { administered } \\
\text { over less than } \\
30 \text { minutes }\end{array}$ & All patients & 60 & Median & & +0 & +2 & +2 & $+0.4 \%$ & -0.2 & $\begin{array}{c}\text { No } \\
\text { change }\end{array}$ & -6 \\
\hline \multirow[t]{2}{*}{$\begin{array}{l}\text { Ospina- } \\
\text { Tascon [57] }\end{array}$} & $\begin{array}{l}400 \mathrm{ml} \text { of } 4 \% \\
\text { albumin or } \\
1,000 \mathrm{ml} \text { of CSL } \\
\text { over } 30 \text { minutes }\end{array}$ & $\begin{array}{l}\text { Patients with } \\
\text { early sepsis }\end{array}$ & 60 & Median & $\begin{array}{l}+300 \mathrm{ml} / \\
\mathrm{min} / \mathrm{m}^{2}\end{array}$ & +2 & +2 & +3 & $+2 \%$ & -0.2 & & \\
\hline & $\begin{array}{l}400 \mathrm{ml} \text { of } 4 \% \\
\text { albumin or } \\
1,000 \mathrm{ml} \text { of CSL } \\
\text { over } 30 \text { minutes }\end{array}$ & $\begin{array}{l}\text { Patients with } \\
\text { late sepsis }\end{array}$ & 60 & Median & $\begin{array}{l}+300 \mathrm{ml} / \\
\mathrm{min} / \mathrm{m}^{2}\end{array}$ & -9 & +7 & +1 & $+1 \%$ & +0.1 & & \\
\hline \multirow[t]{2}{*}{$\begin{array}{l}\text { van Haren } \\
\text { [65] }\end{array}$} & $\begin{array}{l}250 \mathrm{ml} \text { of } 6 \% \\
\text { HES in } 7.2 \% \\
\text { saline over } \\
15 \text { minutes }\end{array}$ & Hypertonic bolus & 60 & Mean & $\begin{array}{l}+400 \mathrm{ml} / \\
\mathrm{min} / \mathrm{m}^{2}\end{array}$ & -11 & +6 & +1 & & -0.3 & & -9 \\
\hline & $\begin{array}{l}500 \mathrm{ml} \text { of } 6 \% \\
\text { HES in } 0.9 \% \\
\text { saline over } \\
15 \text { minutes }\end{array}$ & Isotonic bolus & 60 & Mean & $\begin{array}{l}-300 \mathrm{ml} / \\
\mathrm{min} / \mathrm{m}^{2}\end{array}$ & -1 & +3 & +3 & & -0.1 & & -12 \\
\hline
\end{tabular}


Table 3 Physiological effects grouped by measurement time (Continued)

Haemodynamic indices measured greater than 60 minutes after fluid bolus administration

\begin{tabular}{|c|c|c|c|c|c|c|c|c|c|c|c|}
\hline \multirow[t]{6}{*}{$\begin{array}{l}\text { van Haren } \\
\text { [65] }\end{array}$} & $\begin{array}{l}250 \mathrm{ml} \text { of } 6 \% \\
\text { HES in } 7.2 \% \\
\text { saline over } \\
15 \text { minutes }\end{array}$ & Hypertonic bolus & 120 & Mean & $\begin{array}{l}+300 \mathrm{ml} / \\
\mathrm{ml} / \mathrm{m}^{2}\end{array}$ & -7 & +7 & +2 & 0.0 & +13 & -6 \\
\hline & $\begin{array}{l}500 \mathrm{ml} \text { of } 6 \% \\
\text { HES in } 0.9 \% \\
\text { saline over } \\
15 \text { minutes }\end{array}$ & Isotonic bolus & 120 & Mean & $\begin{array}{l}-300 \mathrm{ml} / \\
\mathrm{min} / \mathrm{m}^{2}\end{array}$ & +0 & +1 & +2 & -0.3 & -30 & -9 \\
\hline & $\begin{array}{l}250 \mathrm{ml} \text { of } 6 \% \\
\text { HES in } 7.2 \% \\
\text { saline over } \\
15 \text { minutes }\end{array}$ & Hypertonic bolus & 180 & Mean & $\begin{array}{l}+100 \mathrm{ml} / \\
\mathrm{min} / \mathrm{m}^{2}\end{array}$ & -3 & +6 & +3 & -0.3 & & -9 \\
\hline & $\begin{array}{l}500 \mathrm{ml} \text { of } 6 \% \\
\text { HES in } 0.9 \% \\
\text { saline over } \\
15 \text { minutes }\end{array}$ & Isotonic bolus & 180 & Mean & $\begin{array}{l}+0 \mathrm{ml} / \\
\mathrm{min} / \mathrm{m}^{2}\end{array}$ & +3 & +5 & +3 & -0.2 & & -6 \\
\hline & $\begin{array}{l}250 \mathrm{ml} \text { of } 6 \% \\
\text { HES in } 7.2 \% \\
\text { saline over } \\
15 \text { minutes }\end{array}$ & Hypertonic bolus & 240 & Mean & $\begin{array}{l}+100 \mathrm{ml} / \\
\mathrm{min} / \mathrm{m}^{2}\end{array}$ & +1 & +3 & +3 & -0.3 & -3 & -8 \\
\hline & $\begin{array}{l}500 \mathrm{ml} \text { of } 6 \% \\
\text { HES in } 0.9 \% \\
\text { saline over } \\
15 \text { minutes }\end{array}$ & Isotonic bolus & 240 & Mean & $\begin{array}{l}-200 \mathrm{ml} / \\
\mathrm{min} / \mathrm{m}^{2}\end{array}$ & +3 & +0 & +3 & -0.2 & -40 & -4 \\
\hline
\end{tabular}

$\mathrm{Cl}$, cardiac index; $\mathrm{CO}$, cardiac output; $\mathrm{CSL}$, compound sodium lactate; FFP, fresh frozen plasma; $\mathrm{HES}$, hydroxyethyl starch; $\mathrm{SVI}$, stroke volume index; $\mathrm{VO}_{2}$, oxygen delivery. 

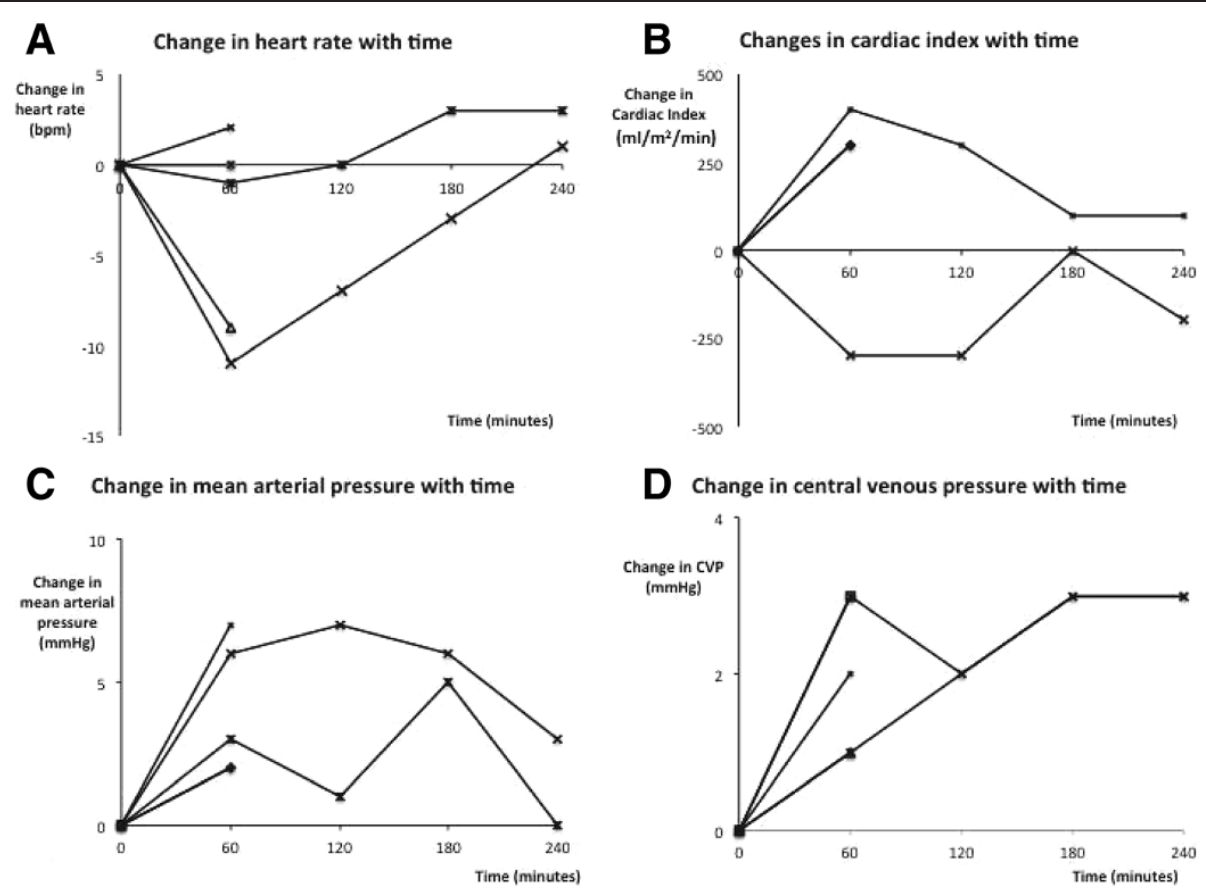

Figure 4 Physiological effects of fluid bolus therapy over time. Multi-panel figure of the haemodynamic effects of fluid bolus therapy (FBT) as reported in studies with observation periods of 60 minutes or more. (A) Changes in heart rate over time. (B) Changes in cardiac index over time. (C) Changes in mean arterial pressure over time. (D) Changes in central venous pressure (CVP) over time. Each solid black line represents a patient group and the average physiological response to FBT over the observation period. Lines terminate when measurements were discontinued in the study from which the group was taken.

changes. We found heterogeneity of triggers, amount, fluid choice and speed of delivery for FBT, which was administered to achieve heterogeneous physiological targets. We similarly found heterogeneity of physiological changes after FBT. In addition, no RCTs compared FBT with an alternative intervention. Finally, no study related physiological changes after FBT to clinically relevant outcomes.

FBT is a widespread intervention in the management of the critically ill septic patient, despite lack of a consistent definition or use of terminology. Our study demonstrates that no contemporary RCTs exist that compare FBT with alternative interventions. The only study comparing FBT to an alternative intervention was a single, non-randomized, prospective, observational study that compared acute circulatory failure patients treated with FBT (500 $\mathrm{ml}$ of saline) or with increased norepinephrine dose according to clinician preference. The two groups had clearly different baseline characteristics and were not directly compared [55]. Alternative interventions to FBT may include a diagnostic low-volume FBT [17], classic fluid challenge [11,12], low-volume FBT and lowdose vasopressor therapy, or cardiac output-guided therapy. Despite the availability of such strategies and the availability of non-invasive cardiac output monitoring, these alternative approaches have not been studied.
Understanding which patient will be fluid responsive is a vital part of rationalising fluid therapy [69]. However, there are multiple different definitions of fluid responsiveness, each dependent on different interventions and different measurements. It would appear that there is little evidence to suggest a consistently different response to FBT based on pre-intervention physiology, as fluid responsiveness is often tautologically and retrospectively defined by participants responses to the therapy. A full review of this topic is beyond the scope of this review, though this information is available elsewhere $[69,70]$.

The contribution of FBT to a positive fluid balance remains poorly understood. In a recent observational study, Bihari and colleagues [36] found that a median of $52.4 \%$ of fluid balance on the first, $30.8 \%$ on the second and $33.2 \%$ on the third study day consisted of FBT. In the Fluid and Catheter Treatment Trial [27] and Sepsis Occurrence in Acutely Ill Patients [71] studies, increasing fluid balance was associated with increased risk of acute kidney injury and mortality. In a retrospective study of septic shock patients in a North American university hospital, non-survivors had a significantly greater positive net fluid balance than survivors over the first 24 hours from onset [34]. Our study also shows little or no evidence for any persisting beneficial physiological changes following FBT. These observations suggest the 
Table 4 Clinically orientated primary outcomes

\begin{tabular}{|c|c|c|c|c|c|c|c|c|c|c|}
\hline First author & Journal & Year & Control group & ICU mortality & $\begin{array}{l}\text { Hospital } \\
\text { mortality }\end{array}$ & Other & Intervention group & $\begin{array}{l}\text { ICU } \\
\text { mortality }\end{array}$ & $\begin{array}{l}\text { Hospital } \\
\text { mortality }\end{array}$ & Other \\
\hline MacRedmond [49] & $\begin{array}{l}\text { Quality and Safety } \\
\text { in Health Care }\end{array}$ & 2010 & $\begin{array}{l}\text { Before protocolised } \\
\text { resuscitation }\end{array}$ & $19 / 37$ & & & After protocolised resuscitation & $10 / 37$ & & \\
\hline Pierrakos [59] & $\begin{array}{l}\text { Intensive Care } \\
\text { Medicine }\end{array}$ & 2012 & $\begin{array}{l}\text { Responders (>10\% } \\
\text { increase in Cl) }\end{array}$ & $13 / 25$ & & & $\begin{array}{l}\text { Non-responders }(<10 \% \\
\text { increase in } \mathrm{Cl})\end{array}$ & $11 / 26$ & & \\
\hline Patel [58] & $\begin{array}{l}\text { Annals of } \\
\text { Pharmacotherapy }\end{array}$ & 2010 & Pre-intervention & & $32 / 53$ & & $\begin{array}{l}\text { Post-intervention, significantly } \\
\text { more fluid and less vasoactives }\end{array}$ & & $12 / 59$ & \\
\hline Castellanos-Ortega [37] & $\begin{array}{l}\text { Critical Care } \\
\text { Medicine }\end{array}$ & 2010 & Pre-intervention & $51 / 96$ & $55 / 96$ & & $\begin{array}{l}\text { Post-intervention, significantly } \\
\text { more fluid }\end{array}$ & $117 / 384$ & $144 / 384$ & \\
\hline Trof [64] & $\begin{array}{l}\text { Critical Care } \\
\text { Medicine }\end{array}$ & 2012 & $\begin{array}{l}\text { Pulmonary artery catheter- } \\
\text { guided resuscitation }\end{array}$ & $13 / 34$ & $15 / 34$ & & $\begin{array}{l}\text { Transpulmonary thermodilution- } \\
\text { guided resuscitation }\end{array}$ & $17 / 38$ & $21 / 38$ & \\
\hline Hanzelka [43] & $\begin{array}{l}\text { Supportive Care in } \\
\text { Cancer }\end{array}$ & 2013 & Pre-intervention & & & 28-day: 38/100 & $\begin{array}{l}\text { Post-intervention, significantly } \\
\text { quicker resuscitation }\end{array}$ & & & 28-day: 20/100 \\
\hline Jacob [44] & $\begin{array}{l}\text { Critical Care } \\
\text { Medicine }\end{array}$ & 2012 & Pre-intervention & & & 30-day: 126/245 & $\begin{array}{l}\text { Post-intervention, significantly } \\
\text { quicker resuscitation with } \\
\text { significantly larger volumes of } \\
\text { fluid at } 6 \text { and } 24 \text { hours }\end{array}$ & & & 30-day: $257 / 426$ \\
\hline
\end{tabular}

$\mathrm{Cl}$, cardiac index. 
Table 5 Clinically orientated secondary outcomes

\begin{tabular}{|c|c|c|c|c|c|c|c|c|c|c|c|c|}
\hline First author & Journal & Year & Control group & $\begin{array}{l}\text { LOS in ICU } \\
\text { (days) }\end{array}$ & $\begin{array}{l}\text { LOS in hospital } \\
\text { (days) }\end{array}$ & $\begin{array}{l}\text { MV } \\
\text { (days) }\end{array}$ & CRRT & Intervention group & $\begin{array}{l}\text { LOS in ICU } \\
\text { (days) }\end{array}$ & $\begin{array}{l}\text { LOS in hospital } \\
\text { (days) }\end{array}$ & $\begin{array}{l}\text { MV } \\
\text { (days) }\end{array}$ & $\overline{\text { CRRT }}$ \\
\hline $\begin{array}{l}\text { MacRedmond } \\
\text { [49] }\end{array}$ & $\begin{array}{l}\text { Quality and Safety in } \\
\text { Health Care }\end{array}$ & 2010 & $\begin{array}{l}\text { Before protocolised } \\
\text { resuscitation }\end{array}$ & 8 & & & & After protocolised resuscitation & 7 & & & \\
\hline $\begin{array}{l}\text { Castellanos- } \\
\text { Ortega [37] }\end{array}$ & Critical Care Medicine & 2010 & Pre-intervention & 9.9 & 26.5 & & & $\begin{array}{l}\text { Intervention group, significantly } \\
\text { more receive fluid }\end{array}$ & 9.1 & 30.6 & & \\
\hline Hanzelka [43] & $\begin{array}{l}\text { Supportive Care in } \\
\text { Cancer }\end{array}$ & 2013 & Pre-intervention & 5.1 & 10.3 & & & $\begin{array}{l}\text { Post-intervention, significantly } \\
\text { quicker resuscitation }\end{array}$ & 2.5 & 8.1 & & \\
\hline Trof [64] & Critical Care Medicine & 2012 & $\begin{array}{l}\text { Pulmonary artery } \\
\text { catheter-guided } \\
\text { resuscitation }\end{array}$ & 15 & 25 & 13 & & $\begin{array}{l}\text { Transpulmonary thermodilution- } \\
\text { guided resuscitation }\end{array}$ & 11 & 27 & 10 & \\
\hline Patel [58] & $\begin{array}{l}\text { Annals of } \\
\text { Pharmacotherapy }\end{array}$ & 2010 & Pre-intervention & 6 & 9.5 & 7.5 & $8 / 53$ & $\begin{array}{l}\text { Post-intervention, significantly } \\
\text { more fluid and less vasoactives }\end{array}$ & 5 & 9 & 7 & $0 / 59$ \\
\hline
\end{tabular}

CRRT, continuous renal replacement therapy; LOS, length of stay; MV, mechanical ventilation. 
need for RCTs comparing FBT with alternative interventions and well-defined triggers and physiological outcomes.

This review has several strengths. To our knowledge this is the first review of the contemporary literature on FBT in critically ill patients with severe sepsis.

We are the first to explore the contemporary features of a FBT, and the first to produce a summary of the physiological changes associated with FBT in septic, critically ill patients, including data from RCTs, and observational and quasi-experimental studies. Our wide search criteria, use of three separate sources and hand searching references reduced the risk of inclusion bias and makes it unlikely that we missed relevant studies.

Our study also has some limitations. Our assessments of physiological changes are necessarily limited to the measures of central tendency provided in tables and graphs in the studies identified. We have only provided crude median results in an attempt to provide a rough estimate of possible effect. We limited our search to the present evolving decade. It is unlikely that current clinical practice is better reflected by earlier studies. Indeed, in comparing our results with similar, earlier studies, the reported physiological changes are similar [14,71-75]. We did not account for the effect of vasoactive medications beyond noting their administration. It appears obvious that the mixed and differential inotropic/vasopressor/lusitropic/ chronotropic effects of different vasoactive medications are likely to have an effect on the physiological changes reported, as would the administration of blood products. Inadequate information was provided in the studies to make such adjustments possible. FBT is normally part of a complex intervention - the resuscitation of the critically ill patient. As well as the initiation and manipulation of vasoactive medications, analyses must contend with the impact of the use of mechanical ventilation, CRRT, and antibiotic administration. These confounders were not reliably reported in the studies identified and could not be evaluated. In addition, the perceived haemodynamic success of an intervention often depends on the trajectory of the patient s clinical course. Unfortunately no such information was available from the studies reviewed.

\section{Conclusion}

FBT in severe sepsis and septic shock is described in 33 articles in the contemporary literature. Only 17 of these studies report the physiological changes associated with FBT. Evidence regarding the efficacy of FBT compared with alternative interventions is lacking. Crucially, no studies relate the physiological changes after FBT to clinically relevant outcomes. In light of recent studies highlighting the association between FBT and fluid administration in general and harm, there is a clear need for at least obtaining randomised controlled evidence for the physiological effects of FBT over the immediate ( 0 to 4 hours) post-intervention period in patients with severe sepsis and septic shock.

\section{Additional file}

Additional file 1: Electronic Supplement. Containing: Appendix 1 (Electronic Search Strategies); Table S1: Study inclusion criteria, definitions of sepsis and definitions of hyperlactataemia; Table S2: Physiological effects grouped by intervention type and comparison; Table S3: Physiological effects grouped by speed of FBT delivery; Table S4: Physiological effects of FBT grouped by fluid class.

\section{Abbreviations}

CRRT: Continuous renal replacement therapy; CVP: Central venous pressure; FBT: Fluid bolus therapy; IV: Intravenous; RCT: Randomised controlled trial.

\section{Competing interests}

The authors declare that they have no competing interests.

\section{Author s contributions}

NJG: study design, electronic search design, literature search, study selection, data extraction, data handling/analysis, manuscript preparation, manuscript revision, and manuscript submission. GME: literature search, study selection, manuscript revision, and manuscript submission. RB: study design, electronic search design, data analysis, manuscript preparation, manuscript revision, and manuscript submission. All authors read and approved the final manuscript.

\section{Author details}

${ }^{1}$ Department of Intensive Care, Austin Hospital, Melbourne, Victoria 3084, Australia. ${ }^{2}$ Australian and New Zealand Intensive Care Research Centre, School of Public Health and Preventive Medicine, Monash University, Melbourne, Victoria 3004, Australia. ${ }^{3}$ School of Nursing and Midwifery, Faculty of Health, Deakin University, Burwood, Victoria 3125, Australia.

Published online: 27 December 2014

\section{References}

1. Holliday MA, Segar WE: The maintenance need for water in parenteral fluid therapy. Pediatrics 1957, 19:823 832.

2. Network SIG: Postoperative management in adults. A practical guide to postoperative care for clinical staff. In SIGN Guidelines. Healthcare Improvement Scotland: Edinburgh; 2004.

3. Powell-Tuck J, Gosling P, Lobo DN, Allison SP, Carlson GL, Gore M, Lewington AJ, Pearse RM, Mythen MG: British consensus guidelines on intravenous fluid therapy for adult surgical patients (GIFTASUP). [http://www.bapen.org.uk/pdfs/bapen_pubs/giftasup.pdf]

4. Resuscitation Council UK: Advanced Life Support Manual. 6th edition. London: Resuscitation Council (UK); 2011.

5. Carey JS, Mohr PA, Brown RS, Shoemaker WC: Cardiovascular function in hemorrhage, trauma and sepsis: determinants of cardiac output and cardiac work. Ann Surg 1969, 170:910 921.

6. Hall JE: Cardiac output, venous return and their regulation. In Guyton and Hall Textbook of Medical Physiology. 12th edition. Edited by Hall JE, Guyton AC. Philadeliphia, PA: Saunders Elsevier; 2011:229 241.

7. Shoemaker WC: Pathophysiologic mechanisms in shock and their therapeutic implications. Am J Surg 1965, 110:337 341.

8. Shoemaker WC, Carey JS, Mohr PA, Brown RS, Monson DO, Yao ST, Kho LK, Stevenson A: Hemodynamic measurements in various types of clinical shock. Analysis of cardiac output and derived calculations in 100 surgical patients. Arch Surg 1966, 93:189 195.

9. Udhoji VN, Weil MH, Sambhi MP, Rosoff L: Hemodynamic studies on clinical shock associated with infection. Am J Med 1963, 34:461 469.

10. Weil MH: Current concepts on the management of shock. Circulation 1957, 16:1097 1105.

11. Vincent JL, Weil MH: Fluid challenge revisited. Crit Care Med 2006, $34: 13331337$ 
12. Weil MH, Henning RJ: New concepts in the diagnosis and fluid treatment of circulatory shock. Thirteenth annual Becton, Dickinson and Company Oscar Schwidetsky Memorial Lecture. Anesth Analg 1979, 58:124 132.

13. Weil MH, Shubin $H$, Rosoff L: Fluid repletion in circulatory shock: central venous pressure and other practical guides. JAMA 1965, 192:668 674.

14. Shoemaker WC: Comparison of the relative effectiveness of whole blood transfusions and various types of fluid therapy in resuscitation. Crit Care Med 1976, 4:71 78

15. Shoemaker WC: Evaluation of colloids, crystalloids, whole blood, and red cell therapy in the critically ill patient. Clin Lab Med 1982, 2:35 63.

16. Shoemaker WC, Hopkins JA, Greenfield S, Chang PC, Umof P, Shabot MM, Spenler CW, State D: Resuscitation algorithm for management of acute emergencies. JACEP 1978, 7:361 367.

17. Muller L, Toumi M, Bousquet PJ, Riu-Poulenc B, Louart G, Candela D, Zoric L, Suehs C, de La Coussaye JE, Molinari N, Lefrant JY, AzuRa Group: An increase in aortic blood flow after an infusion of $100 \mathrm{ml}$ colloid over 1 minute can predict fluid responsiveness: the mini-fluid challenge study. Anesthesiology 2011, 115:541 547

18. Dellinger RP, Carlet JM, Masur H, Gerlach H, Calandra T, Cohen J, GeaBanacloche J, Keh D, Marshall JC, Parker MM, Ramsay G, Zimmerman JL, Vincent $J$, Levy MM: Surviving Sepsis Campaign guidelines for management of severe sepsis and septic shock. Intensive Care Med 2004, 30:536 555.

19. Dellinger RP, Levy MM, Carlet JM, Bion J, Parker MM, Jaeschke R, Reinhart K, Angus DC, Brun-Buisson C, Beale R, Calandra T, Dhainaut JF, Gerlach H, Harvey M, Marini JJ, Marshall J, Ranieri M, Ramsay G, Sevransky J, Thompson BT, Townsend S, Vender JS, Zimmerman JL, Vincent JL, International Surviving Sepsis Campaign Guidelines Committee; American Association of Critical-Care Nurses; American College of Chest Physicians; American College of Emergency Physicians; Canadian Critical Care Society; European Society of Clinical Microbiology and Infectious Diseases, et al: Surviving Sepsis Campaign: international guidelines for management of severe sepsis and septic shock: 200. Crit Care Med 2008, 36:296 327

20. Dellinger RP, Levy MM, Rhodes A, Annane D, Gerlach H, Opal SM, Sevransky JE, Sprung CL, Douglas IS, Jaeschke R, Osborn TM, Nunnally ME, Townsend SR, Reinhart K, Kleinpell RM, Angus DC, Deutschman CS, Machado FR, Rubenfeld GD, Webb SA, Beale RJ, Vincent JL, Moreno R, Surviving Sepsis Campaign Guidelines Committee including the Pediatric Subgroup: Surviving Sepsis Campaign: International guidelines for management of severe sepsis and septic shock: 2012. Crit Care Med 2013, 41:580 637.

21. McLuckie A: Shock - an overview. In Ohs Intensive Care Manual. 6th edition. Edited by Bersten AD. Philadelphia: Elsevier Limited; 2009:97 104.

22. Hilton AK, Bellomo R: A critique of fluid bolus resuscitation in severe sepsis. Crit Care 2012, 16:302.

23. Axler O, Tousignant C, Thompson CR, Dalla va-Santucci J, Drummond A, Phang PT, Russell JA, Walley KR: Small hemodynamic effect of typical rapid volume infusions in critically ill patients. Crit Care Med 1997, 25:965 970.

24. Bouchard J, Soroko SB, Chertow GM, Himmelfarb J, Ikizler TA, Paganini EP, Mehta RL, Program to Improve Care in Acute Renal Disease (PICARD) Study Group: Fluid accumulation, survival and recovery of kidney function in critically ill patients with acute kidney injury. Kidney Int 2009, 76:422 427.

25. Finfer S, Bellomo R, Boyce N, French J, Myburgh J, Norton R, Safe Study Investigators: A comparison of albumin and saline for fluid resuscitation in the intensive care unit. N Engl J Med 2004, 350:2247 2256.

26. Finfer S, McEvoy S, Bellomo R, McArthur C, Myburgh J, Norton R: Impact of albumin compared to saline on organ function and mortality of patients with severe sepsis. Intensive Care Med 2011, 37:86 96

27. Grams ME, Estrella MM, Coresh J, Brower RG, Liu KD, National Heart, Lung, and Blood Institute Acute Respiratory Distress Syndrome Network: Fluid balance, diuretic use, and mortality in acute kidney injury. Clin J Am Soc Nephrol 2011, 6:966 973 .

28. Heart N, Lung, and Blood Institute Acute Respiratory Distress Syndrome (ARDS) Clinical Trials Network, Wiedemann HP, Wheeler AP, Bernard GR, Thompson BT, Hayden D, deBoisblanc B, Connors AF Jr, Hite RD, Harabin $\mathrm{AL}$ : Comparison of two fluid-management strategies in acute lung injury. N Engl J Med 2006, 354:2564 2575.

29. Berg S, Engman A, Hesselvik JF, Laurent TC: Crystalloid infusion increases plasma hyaluronan. Crit Care Med 1994, 22:1563 1567.

30. Berg S, Golster M, Lisander B: Albumin extravasation and tissue washout of hyaluronan after plasma volume expansion with crystalloid or hypooncotic colloid solutions. Acta Anaesthesiol Scand 2002, 46:166 172
31. Steppan J, Hofer S, Funke B, Brenner T, Henrich M, Martin E, Weitz J, Hofmann U, Weigand MA: Sepsis and major abdominal surgery lead to flaking of the endothelial glycocalix. J Surg Res 2011, 165:136 141.

32. Burke-Gaffney A, Evans TW: Lest we forget the endothelial glycocalyx in sepsis. Crit Care 2012, 16:121

33. Woodcock TE, Woodcock TM: Revised Starling equation and the glycocalyx model of transvascular fluid exchange: an improved paradigm for prescribing intravenous fluid therapy. Br J Anaesth 2012, 108:384 394.

34. Hilton AK, Bellomo R: Totem and taboo: fluids in sepsis. Crit Care 2011 15:164.

35. Reade MC, Huang DT, Bell D, Coats TJ, Cross AM, Moran JL, Peake SL, Singer M, Yealy DM, Angus DC, British Association for Emergency Medicine; UK Intensive Care Society; UK Society for Acute Medicine; Australasian Resuscitation in Sepsis Evaluation Investigators; Protocolized Care for Early Septic Shock Investigators: Variability in management of early severe sepsis. Emerg Med J 2010, 27:110 115

36. Bihari S, Prakash S, Bersten AD: Post resusicitation fluid boluses in severe sepsis or septic shock: prevalence and efficacy (price study). Shock 2013 40:28 34

37. Castellanos-Ortega A, Suberviola B, Garcia-Astudillo LA, Holanda MS, Ortiz F, Llorca J, Delgado-Rodriguez M: Impact of the Surviving Sepsis Campaign protocols on hospital length of stay and mortality in septic shock patients: results of a three-year follow-up quasi-experimental study. Crit Care Med 2010, 38:1036 1043

38. De Backer D, Biston P, Devriendt J, Madl C, Chochrad D, Aldecoa C, Brasseur A, Defrance P, Gottignies P, Vincent JL, SOAP II Investigators: Comparison of dopamine and norepinephrine in the treatment of shock. N Engl J Med 2010, 362:779 789

39. Dong ZZ, Fang Q, Zheng X, Shi H: Passive leg raising as an indicator of fluid responsiveness in patients with severe sepsis. World J Emerg Med 2012, 3:191 196

40. Freitas FGR, Bafi AT, Nascente APM, Assuncao M, Mazza B, Azevedo LCP, Machado FR, Mahajan RP: Predictive value of pulse pressure variation for fluid responsiveness in septic patients using lung-protective ventilation strategies. Br J Anaesth 2013, 110:402 408.

41. Gaieski DF, Mikkelsen ME, Band RA, Pines JM, Massone R, Furia FF, Shofer FS, Goyal M: Impact of time to antibiotics on survival in patients with severe sepsis or septic shock in whom early goal-directed therapy was initiated in the emergency department. Crit Care Med 2010, 38:1045 1053.

42. Hamzaoui O, Georger JF, Monnet X, Ksouri H, Maizel J, Richard C, Teboul JL: Early administration of norepinephrine increases cardiac preload and cardiac output in septic patients with life-threatening hypotension. Crit Care 2010, 14:R142

43. Hanzelka KM, Yeung SC, Chisholm G, Merriman KW, Gaeta S, Malik I, Rice TW: Implementation of modified early-goal directed therapy for sepsis in the emergency center of a comprehensive cancer center. Support Care Cancer 2013, 21:727 734

44. Jacob ST, Banura P, Baeten JM, Moore CC, Meya D, Nakiyingi L, Burke R, Horton CL, Iga B, Wald A, Reynolds SJ, Mayanja-Kizza H, Scheld WM, Promoting Resource-Limited Interventions for Sepsis Management in Uganda Study Group: The impact of early monitored management on survival in hospitalized adult Ugandan patients with severe sepsis: a prospective intervention study. Crit Care Med 2012, 40:2050 2058.

45. Khwannimit B, Bhurayanontachai R: Prediction of fluid responsiveness in septic shock patients: comparing stroke volume variation by FloTrac/Nigileo and automated pulse pressure variation. Eur J Anaesthesiol 2012, 29:64 69.

46. Lakhal K, Ehrmann S, Perrotin D, Wolff M, Boulain T: Fluid challenge: tracking changes in cardiac output with blood pressure monitoring (invasive or non-invasive). Intensive Care Med 2013, 39:1953 1962.

47. Lanspa MJ, Brown SM, Hirshberg EL, Jones JP, Grissom CK: Central venous pressure and shock index predict lack of hemodynamic response to volume expansion in septic shock: a prospective, observational study. J Crit Care 2012, 27:609 615.

48. Machare-Delgado E, Decaro M, Marik PE: Inferior vena cava variation compared to pulse contour analysis as predictors of fluid responsiveness: a prospective cohort study. J Intensive Care Med 2011, 26:116 124.

49. MacRedmond R, Hollohan K, Stenstrom R, Nebre R, Jaswal D, Dodek P. Introduction of a comprehensive management protocol for severe sepsis is associated with sustained improvements in timeliness of care and survival. Qual Saf Health Care 2010, 19:e46. 
50. Mahjoub Y, Benoit-Fallet H, Airapetian N, Lorne E, Levrard M, Seydi AA, Amennouche $\mathrm{N}$, Slama M, Dupont $\mathrm{H}$ : Improvement of left ventricular relaxation as assessed by tissue Doppler imaging in fluid-responsive critically ill septic patients. Intensive Care Med 2012, 38:1461 1470.

51. McIntyre LA, Fergusson DA, Cook DJ, Rowe BH, Bagshaw SM, Easton D, Emond M, Finfer S, Fox-Robichaud A, Gaudert C, Green R, Hebert P, Marshall J, Rankin N, Stiell I, Tinmouth A, Pagliarello J, Turgeon AF, Worster A, Zarychanski R, Canadian Critical Care Trials Group: Fluid resuscitation with $5 \%$ albumin versus normal saline in early septic shock: a pilot randomized, controlled trial. J Crit Care 2012, 27:317.e311 317.e316.

52. Monnet $X$, Anguel N, Naudin B, Jabot J, Richard C, Teboul J: Arterial pressure-based cardiac output in septic patients: different accuracy of pulse contour and uncalibrated pressure waveform devices. Crit Care 2010, 14:R109

53. Monnet X, Jabot J, Maizel J, Richard C, Teboul JL: Norepinephrine increases cardiac preload and reduces preload dependency assessed by passive leg raising in septic shock patients. Crit Care Med 2011, 39:689 694.

54. Monnet X, Julien F, Ait-Hamou N, Lequoy M, Gosset C, Jozwiak M, Persichini $\mathrm{R}$, Anguel N, Richard C, Teboul JL: Lactate and venoarterial carbon dioxide difference/arterial-venous oxygen difference ratio, but not central venous oxygen saturation, predict increase in oxygen consumption in fluid responders. Crit Care Med 2013, 41:1412 1420.

55. Monnet X, Letierce A, Hamzaoui O, Chemla D, Anguel N, Osman D, Richard C, Teboul $J$ : Arterial pressure allows monitoring the changes in cardiac output induced by volume expansion but not by norepinephrine. Crit Care Med 2011, 39:1394 1399.

56. O Neill R, Morales J, Jule M: Early goal-directed therapy (EGDT) for severe sepsis/septic shock: which components of treatment are more difficult to implement in a community-based emergency department? J Emerg Med 2012, 42:503 510.

57. Ospina-Tascon G, Neves AP, Occhipinti G, Donadello K, Bchele G, Simion D, Chierego ML, Silva TO, Fonseca A, Vincent JL, De Backer D: Effects of fluids on microvascular perfusion in patients with severe sepsis. Intensive Care Med 2010, 36:949 955

58. Patel GW, Roderman N, Gehring H, Saad J, Bartek W: Assessing the effect of the Surviving Sepsis Campaign treatment guidelines on clinical outcomes in a community hospital. Ann Pharmacother 2010, 44:1733 1738

59. Pierrakos C, Velissaris D, Scolletta S, Heenen S, De Backer D, Vincent JL: Can changes in arterial pressure be used to detect changes in cardiac index during fluid challenge in patients with septic shock? Intensive Care Med 2012, 38:422 428.

60. Pottecher J, Deruddre S, Teboul JL, Georger JF, Laplace C, Benhamou D, Vicaut $E$, Duranteau J: Both passive leg raising and intravascular volume expansion improve sublingual microcirculatory perfusion in severe sepsis and septic shock patients. Intensive Care Med 2010, 36:1867 1874.

61. Sanchez M, Jimenez-Lendinez M, Cidoncha M, Asensio MJ, Herrero E, Collado A, Santacruz M: Comparison of fluid compartments and fluid responsiveness in septic and non-septic patients. Anaesth Intensive Care 2011, 39:1022 1029.

62. Schnell D, Camous L, Guyomarch S, Duranteau J, Canet E, Gery P, Dumenil AS, Zeni F, Azoulay E, Darmon M: Renal perfusion assessment by renal Doppler during fluid challenge in sepsis. Crit Care Med 2013, 41:1214 1220

63. Sturgess DJ, Pascoe RLS, Scalia G, Venkatesh B: A comparison of transcutaneous Doppler corrected flow time, b-type natriuretic peptide and central venous pressure as predictors of fluid responsiveness in septic shock: a preliminary evaluation. Anaesth Intensive Care 2010, 38:336 341 .

64. Trof RJ, Beishuizen A, Cornet AD, Wit RJ, Girbes AR, Groeneveld AB: Volume-limited versus pressure-limited hemodynamic management in septic and nonseptic shock. Crit Care Med 2012, 40:1177 1185.

65. van Haren FMP, Sleigh J, Boerma EC, La Pine M, Bahr M, Pickkers P, Van Der Hoeven JG: Hypertonic fluid administration in patients with septic shock: a prospective randomized controlled pilot study. Shock 2012, 37:268 275.

66. Wacharasint P, Lertamornpong A, Wattanathum A, Wongsa A: Predicting fluid responsiveness in septic shock patients by using 3 dynamic indices: is it all equally effective? J Med Assoc Thai 2012, 95:S149 S156.

67. Yu M, Pei K, Moran S, Edwards KD, Domingo S, Steinemann S, Ghows M, Takiguchi S, Tan A, Lurie F, Takanishi D Jr: A prospective randomized trial using blood volume analysis in addition to pulmonary artery catheter, compared with pulmonary artery catheter alone, to guide shock resuscitation in critically ill surgical patients. Shock 2011, 35:220 228.

68. Zhang Z, Lu B, Ni H, Sheng X, Jin N: Prediction of pulmonary edema by plasma protein levels in patients with sepsis. J Crit Care 2012, 27:623 629.

69. Marik PE, Lemson J: Fluid responsiveness: an evolution of our understanding. $\mathrm{Br}$ J Anaesth 2014, 112:617 620.

70. Marik PE, Cavallazzi R: Does the central venous pressure predict fluid responsiveness? An updated meta-analysis and a plea for some common sense. Crit Care Med 2013, 41:1774 1781.

71. Payen D, de Pont AC, Sakr Y, Spies C, Reinhart K, Vincent JL: Sepsis Occurrence in Acutely III Patients Investigators: A positive fluid balance is associated with a worse outcome in patients with acute renal failure. Crit Care 2008, 12:R74.

72. Calvin JE, Driedger AA, Sibbald WJ: The hemodynamic effect of rapid fluid infusion in critically ill patients. Surgery 1981, 90:61 76.

73. Perner A, Faber T: Stroke volume variation does not predict fluid responsiveness in patients with septic shock on pressure support ventilation. Acta Anaesthesiol Scand 2006, 50:1068 1073.

74. Sakka SG, Meier-Hellmann A, Reinhart K: Do fluid administration and reduction in norepinephrine dose improve global and splanchnic haemodynamics? Br J Anaesth 2000, 84:758 762.

75. Shoemaker WC, Schluchter M, Hopkins JA, Appel PL, Schwartz S, Chang PC Comparison of the relative effectiveness of colloids and crystalloids in emergency resuscitation. Am J Surg 1981, 142:73 84.

doi:10.1186/s13054-014-0696-5

Cite this article as: Glassford et al:: Physiological changes after fluid bolus therapy in sepsis: a systematic review of contemporary data. Critical Care 2014 18:696. 